

$$
\begin{aligned}
& \text { ONo } 288 \text { DEPARTMENT OF } \\
& \text { FP } 18 \text { 1962 } \\
& \text { intis Industrial University, } \\
& \text { CIIAMPAIGN, . . . . ILLINOIS } \\
& \text { Books are Not to be Taken From the Library Room. }
\end{aligned}
$$





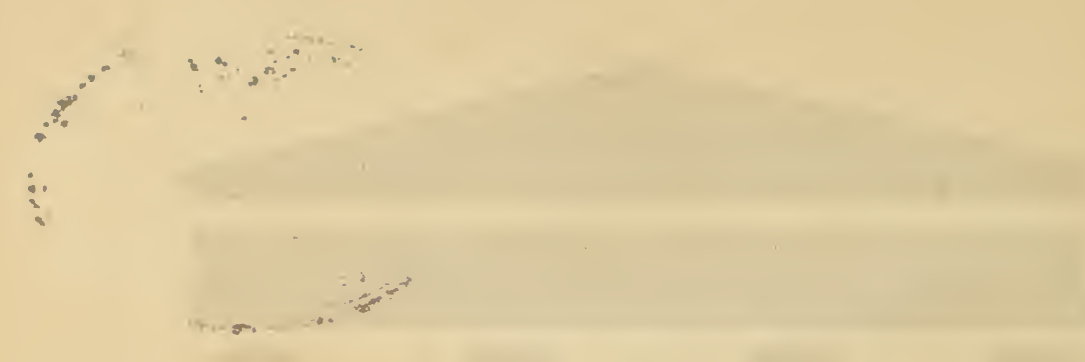

Digitized by the Internet Archive in 2016

https://archive.org/details/indexfungorumbri00cook 


\section{N D E X}

\section{FUNGORUM BRITANNICORUM.}

A COMPLETE LIST

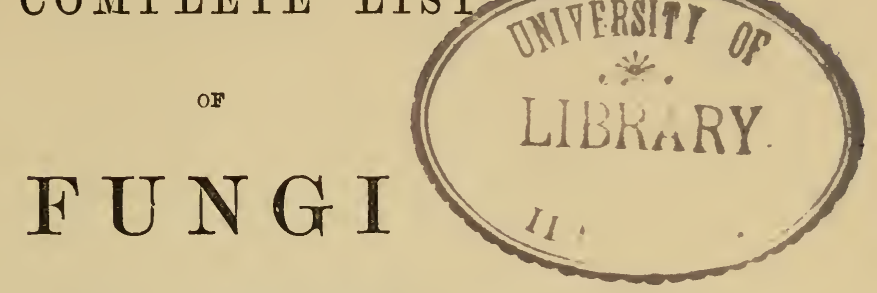

FOUND IN THE BRITISH ISLANDS

TO THE PRESENT DATE,

ARRANGED SO AS TO BE APPLICABLE EITHER AS A CHECK-LIST OR FOR HERBARIUM LABELS.

Br M. C. $\dot{\mathrm{C} O O K \mathrm{~K}}$,

AUTHOR OF "A PLAIN AND EASY ACCOUNT OF BRITISH FUNGI," Etrc.

L O N D O :

ROBERT HARDWICKE, 192, PICCADILLY. 
$589^{2}$

77 in

\section{N O T E.}

It has been considered advisable not to enlarge the present work so as to include the Synonymy. Should the student experience any difficulty on this account, he may be relieved by consulting "Streinz's Nomenclator Fungorum" (Vienna, 1862), in which the majority of the Synonyms may be found.

M. C. C. 


\section{INDEX FUNGORUM BRITANNICORUM.}

\section{FAMILY I.-HYIMENOMYCETMS.}

\section{Order I.-AGARICINI.}

GENUS I.-AGARICUS, $L$.

A.-Leucospori.

Sub-Genus 1.-AMANITA.

1. (Amanita) vernus, Bull.

2. (Amanita) Phalloides, Fr.

3. (Amanita) muscarius, $L$.

4. (Amanita) pantherinus, $D C$.

5. (Amanita) strobiliformis, $F r$.

6. (Amanita) Mappa, Batsch.

7. (Amanita) rubescens, $P$.

8. (Amanita) excelsus, Fr.

9. (Amanita) asper, $\mathrm{Fr}$.

10. (Amanita) megalodactylus, $B$.

11. (Amanita) vaginatus, Bull.

12. (Amanita) Ceciliæ, B.\&Br.

Sub-Genus 2.-LEPIOTA.

13. (Lepiota) procerus, Scop.

14. (Lepiota) rachodes, Vitt.

15. (Lepiota) excoriatus, Schaff.

16. (Lepiota) gracilentus, Kromb.
17. (Lepiota) mastoideus, Fr.

18. (Lepiota) acutesquamosus, $W m$.

19. (Lepiota) hispidus, Lasch.

20. (Lepiota) Badhami, B.\&Br.

21. (Lepiota) clypeolarius, Bull.

22. (Lepiota) cristatus, Fr.

23. (Lepiota) Vittadini, Moretti.

24. (Lepiota) naucinus, $F r$.

25. (Lepiota) cepæstipes, Sow.

26. (Lepiota) granulosus, Batsch. var. amianthinus, Scop.

27. (Lepiota) polystictus, Berk.

28. (Lepiota) gliodermus, $F r$.

Sub-Genus 3.-ARMILLARIA.

29. (Armillaria) ramentaceus, Bull.

30. (Armillaria) constrictus, $F r$.

31. (Armillaria) melleus, Vahl.

32. (Armillaria) mucidus, $F r$.

Sub-Genus 4. -TRICHOLOMA.

33. ('Tricholoma) equestris, Linn.

34. (Tricholoma) sejunctus, Sow. 
35. (Tricholoma) portentosus, Fr. 36. (Tricholoma) fucatus, Fr.

37. ('Tricholoma) spermaticus, $F r$. 38. (Tricholoma) nictitans, $F r$. 39. (Tricholoma) fulvollus, $F r$. 40. (Tricholoma)flavo-brunneus, $I \%$. 41. (Tricholoma) albo-brunneus, $P$. 42. (Tricholoma) rutilans, Schceff. 43. (Tricholoma) luridus, Schaff. 44. (Tricholoma) Columbetta, $F r$. 45. (Tricholoma) imbricatus, Fr. 46. (Tricholoma) vaccinus, $P$.

47. (Tricholoma) crassifolius, Berk. 48. (Tricholoma) murinaceus, Bull. 49. (Tricholoma) terreus, Schaff.

50. (Tricholoma) scalpturatus, $F i$. 51. (Tricholoma) saponaceus, Fr.

52. (Tricholoma) meleagris, Souv.

53. (Tricholoma) cartilagineus, Bull.

54. (Tricholoma) cuneifolius, $\mathrm{Fr}$. 55. (Tricholoma) sulfureus, Bull. 56. (Tricholoma) bufonius, $P$. 57. (Tricholoma) lascivus, $F r$. 58. (Tricholoma) inamœnus, Fr. 59. (Tricholoma) immundus, Bert. 60. (Tricholoma) ionides, Bull. 61. (Tricholoma) carneus, Bull. 62. (Tricholoma) gambosus, $\mathrm{Fr}$. 63. (Tricholoma) monstrosus, Sow. 64. (Tricholoma) albus, Fr.

65. (Tricholoma) personatus, Fr. 66. (Tricholoma) nudus, Bull. 67. (Tricholoma) acerbus, Bull. 68. (Tricholoma) cinerascens, Bull
69. (Tricholoma) grammopodius, Bull.

70. (Tricholoma) melaleucus, $P$.

71. (Tricholoma) humilis, $F r$.

72. (Tricholoma) subpulverulentus, $P$.

Sub-Genus 5.-CLITOCYBE.

73. (Clitocybe) nebularis, Batsch.

74. (Clitocybo) fumosus, $P$.

75. (Clitocybe) inornatus, Sow.

76. (Clitocybe) odorus, Bull.

77. (Clitocybe) cerussatus, Fr.

78. (Clitocybe) phyllophilus, Fr.

79. (Clitocybe) candicans, Fr.

80. (Clitocybe) dealbatus, $P$.

81. (Clitocybe) gallinaceus, Scop.

82. (Clitocybe) elixus, Sow.

83. (Clitocybe) opacus, With.

84. (Clitocybe) giganteus, Sow.

85. (Clitocybe) infundibuliformis, Schaff.

86. (Clitocybe) geotrupus, Bull.

87. (Clitocybe) inversus, Scop.

88. (Clitocybe) flaccidus, Sow.

89. (Clitocybe) cyathiformis, Fr.

90. (Clitocybe) brumalis, Fr.

91. (Clitocybe) metachrous, $\mathrm{Fr}$.

92. (Clitocybe) fragrans, Sow.

93. (Clitocybe) difformis, $P$.

94. (Clitocybe) ectypus, $\mathrm{Fr}$.

95. (Clitocybe) bellus, $P$.

96. (Clitocybe) laccatus, Scop.

Sub-Genus 6.-COLLYBIA.

97. (Collybia) radicatus, Relh.

98. (Collybia) longipes, Bull. 
99. (Collybia) platyphyllus, $\mathrm{Fr}$.

100. (Collybia) fusipes, Bull.

101. (Collybia) maculatus, $A$. \& $S$.

102. (Collybia) butyraceus, Bull.

103. (Collybia) velutipes, Curt.

104. (Collybia) stipitarius, Fr.

105. (Collybia) confluens, $P$.

106. (Collybia) ingratus, Schum.

107. (Collybia) undatus, Berk.

108. (Collybia) conigenus, $P$.

109. (Collybia) cirrhatus, Schum.

110. (Collybia) tuberosus, Bull.

111. (Collybia) racemosus, $P$.

112. (Collybia) xanthopus, $F r$.

113. (Collybia) esculentus, Jacq.

114. (Collybia) tenacellus, $P$.

115. (Collybia) acervatus, $\mathrm{Fr}$.

116. (Collybia) dryophilus, Bull.

117. (Collybia) exsculptus, $F r$.

118. (Collybia) clavus, Bull.

119. (Collybia) ocellatus, Fr.

120. (Collybia) laceratus, Lasch.

121. (Collybia) atratus, $F r$.

Sub-Genus 7.-MYCENA.

122. (Mycena) pelianthinus, Fr.

123: (IIycena) balaninus, $P$.

124. (INycena) elegans, $P$.

125. (Mycena)rubromarginatus.Fr.

126. (Mycena) strobilinus, Sow.

127. (Mycena) rosellus, $\mathrm{Fr}$.

128. (Mycena) purus, $P$.

129. (Mycena) Iris, Berk.

130. (Mycena) Adonis, Bull.

131. (Mycena) luteo-albus, Bolt.

132. (IMycena) lacteus, $P$.
133. (IMycena) proliferus, Sow.

134. (Mycena) galericulatus, Scop.

135. (Mycena) polygrammus, Bull.

136. (IIycena) parabolicus, A.\&S.

137. (Mycena) atro-albus, Bull.

138. (IIycena) dissiliens, $F r$.

139. (Nycena) alcalinus, $F r$.

140. (Mycena) pauperculus, Berk.

141. (Mycena) tenuis, Bolt.

142. (Mycena) filopes, Bull.

143. (Mycena) vitilis, $F r$.

144. (Mycena) speireus, Fr.

145. (Mijcena) acicula, Schaff.

146. (Mycena) sanguineolentus, A. \& $S$.

147. (Mycena) crocatus, Schrad.

148. (Mycena) galopus, Schrad.

149. (Mycena) epipterygius, Scop.

150. (Mycena) pelliculosus, $\mathrm{Ir}$.

151. (Mycena) vulgaris, $P$.

152. (Mycena) roridus, $F r$.

153. (Mycena) stylobates, $P$.

154. (Mycena) tenerrimus, $B$.

155. (Mycena) pterigenus, $F r$.

156. (MIycena) corticola, Schum.

157. (Mycena) setosus, Sow.

158. (Mycena) capillaris, Schum.

159. (IIycena) juncicola, Fr.

Sub-Genus 8.-OMPHALIA.

160. (Omphalia) pyxidatus, Bull.

161. (Omphalia) hepaticus, Batsch.

162. (Omphalia) sphagnicola, Berk.

163. (Omphalia) muralis, Sow.

164. (Omphalia) umbelliferus, $L$.

165. (Omphalia) Felvelloides, Bull. 
166. (Omphalia) rufulus, $B . \& B r$.

167. (Omphalia) stellatus, Sow.

168. (Omphalia) Campanolla, I3atsch.

169. (Omphalia) camptophyllus, Berk.

170. (Omphalia) griseus, $F r$.

171. (Omphalia) Fibula, Bull.

172. (Omphalia) Belliæ, Jolınst.

173. (Omphalia) integrellus, $P$.

Sub-Genus 9.-PLEUROTUS. 174. (Pleurotus) dryinus, $P$.

175. (Pleurotus) ulmarius, Bull.

176. (Pleurotus) subpalmatus, $F r$.

177. (Pleurotus) fimbriatus, Bolt.

178. (Pleurotus) euosmus, Berk.

179. (Pleurotus) ostreatus, Jacq.

180. (Pleurotus) salignus, Hoff $m$.

181. (Pleurotus) petaloides, Bull.

182. (Pleurotus) serotinus, Sclirad.

183. (Pleurotus) mitis, $P$.

184. (Pleurotus) tremulus, Schaff.

185. (Pleurotus) acerosus, $F r$.

186. (Pleurotus) porrigens, $P$.

187. (Pleurotus) septicus, $P$.

188. (Pleurotus) mastrucatus, $F r$.

189. (Pleurotus) atrocæruleus, $F r$.

190. (Pleurotus) algidus, $F r$.

191. (Pleurotus) Leightoni, Berk.

102. (Pleurotus) cyphellæformis, Berk.

193. (Pleurotus) Hobsoni, Berk.

194. (Pleurotus) applicatus, Batsch.

195. (Pleurotus) striatulus, $F r$.

196. (Pleurotus) hypnophilus, $P$.

197. (Pleurotus) chioneus, $P$.
13.-HYPORIIODII.

Sul-Genus 10.-VOLVARIA.

198. (Volvaria) bombycinus, Sclia.ff.

199. (Volvaria) volvaceus, Bull.

200. (Volvaria) Loveianus, Berk.

201. (Volvaria) Taylori, Berk.

202. (Volvaria) parvulus, Weinm.

203. (Volvaria) speciosus, Fr.

204. (Volvaria) gloiocephalus, Fr.

Sub-Genus 11.-PLUTEUS.

205. (Pluteus) cervinus, Schaff.

206. (Pluteus) umbrinus, $F r$.

207. (Pluteus) nanus, $P$.

208. (Pluteus) petasatus, Fr.

209. (Pluteus) leoninus, Schaff.

210. (Pluteus) chrysophæus,

Schaff.

211. (Pluteus) phlebophorus, Ditm.

Sub-Genus 12.-ENTOLOMA.

212. (Entoloma) fertilis, $P$.

213. (Entoloma) prunuloides, Fr.

214. (Entoloma) Placenta, Batsch.

215. (Entoloma) Elodes, $\mathrm{Fr}$.

216. (Entoloma) repandus, Bull.

217. (Entoloma) Bloxami, B.\& Br.

218. (Entoloma) frumentaceus,

Bull.

219. (Fntoloma) sericellus, $F r$.

220. (Entoloma) clypeatus, $L$.

221. (Entoloma) rhodopolius, $F r$.

222. (Entoloma) costatus, $F r$.

223. (Entoloma) sericeus, Bull.

224. (Entoloma) nidorosus, $F r$. 
Sub-Genus 13.-CLITOPILUS. 225. (Clitopilus) prunulus, Scop. 226. (Clitopilus) cretatus, Berl. 227. (Clitopilus) mundulus, Lasch.

Sub-Genus 14.-LEPTONIA. 228. (Leptonia) lampropus, $F r$. 229. (Leptonia) serrulatus, $P$. 230. (Leptonia) euchrous, $P$. 231. (Leptonia) chalybæus, $P$. 232. (Leptonia) incanus, $F r$. 233. (Leptonia) asprellus, Fr.

Sub-Genus 15.-NOLANEA. 234. (Nolanea) pascuus, $P$. 235. (Nolanea) rufo-carneus, Berk. 236. (Nolanea) rubidus, Berk. 237. (Nolanea) Babingtonii, Blox.

Sub-Genus 16.-ECCILIA. 238. (Eccilia) rhodocalyx, Lasch.

$$
\text { (1.-DERMini. }
$$

Sub-Genus 17.-PHOLIOTA. 239. (Pholiota) durus, Bolt. 240. (Pholiota) præcox, $P$. 241. (Pholiota) radicosus, Bull. 242. (Pholiota) pudicus, Bull. 243. (Pholiota) leochromus, Cooke. 244. (Pholiota) capistratus, Cooke. 245. (Pholiota) aurivellus, Batsch. 246. (Pholiota) squarrosus, MIüll. 247. (Pholiota) spectabilis, $F r$. 248. (Pholiota) adiposus, Fr. 249. (Pholiota) flammans, $F r$. 250. (Pholiota) mutabilis, Schaff. 251. (Pholiota) marginatus, Batsch.
252. (Pholiota) pumilus, $F r$.

253. (Pholiota) mycenoides, $F r$. 254. (Pholiota) Leveillianus, D. \& $M$.

Sub-Genus 18.-HEBELOMA. 255. (Hebeloma) relicinus, Fr. 256. (Hebeloma) lanuginosus, $\mathrm{Fr}$. 257. (Hebeloma) plumosus, Bolt. 258. (Hebeloma) pyriodorus, $P$. 259. (Hebeloma) scaber, IIüll. 260. (Hebeloma) lacerus, $F r$. 261. (Hebeloma) flocculosus, Berk. 262. (Hebeloma) Hookeri, Klotsch. 263. (Hebeloma) obscurus, $P$. 264. (Hebeloma) fibrosus, Sow. 265. (Hebeloma) fastigiatus, $\mathrm{Fr}$. 266. (Hebeloma) Curreyi, Berk. 267. (Hebeloma) rimosus, Bull. 268. (Hebeloma) auricomus, Batsch. 269. (Hebeloma) trechisporus, Berk. 270. (Hebeloma) sindonius, $F r$. 271. (Hebeloma) geophyllus, Sow. 272. (Hebeloma) lucifugus, $F r$. 273. (Hebeloma) fastibilis, $F r$. 274. (Hebeloma) testaceus, Batsch. 275. (Hebeloma) punctatus, Fr. 276. (Hebeloma) versipellis, $\mathrm{Fr}$. 277. (Hebeloma) mesophæus, Pers. 278. (Hebeloma) crustuliniformis, Bull.

279. (Hebeloma) longicaudus, $P$.

Sub-Genus 19.-FLAMMULA. 2\&0. (Flammula) scambus, $F r$. 281. (Flammula) flocifer, Bert. 
282. (Flammula) lentus, $P$.

283. (Flammula) flavidus, Schaff.

284. (Flammula) hybridus, $F r$.

285. (Flammula) filiceus, Cooke.

Sub-Genus 20.-NAUCORIA.

286. (Naucoria) cucumis, $P$.

287. (Naucoria) centunculus, $F r$.

288. (Naucoria) horizontalis, Bull.

289. (Naucoria) melinoides, $F r$.

290. (Naucoria) vervacti, Fr.

291. (Naucoria) pediades, $F r$.

292. (Naucoria) semiorbicularis, Bull.

293. (Naucoria) inquilinus, $F r$.

294. (Naucoria) furfuraceus, $P$.

295. (Naucoria) sobrius, Fr.

296. (Naucoria) erinaceus, $F r$.

297. (Naucoria) siparius, Fr.

298. (Naucoria) conspersus, Pers.

299. (Naucoria) carpophilus, $F r$.

Sub-Genus 21.-GALERA.

300. (Galera) reticulatus, $P$.

301. (Galera) lateritius, $F r$.

302. (Galera) tener, Schoeff.

303. (Galera) ovalis, Fr.

304. (Galera) confertus, Bolt.

305. (Galera) sparteus, Fr.

306. (Galera) embolus, $F r$.

307. (Galera) Hypnorum, Batsch.

Sub-Genus 22.-CREPIDOTUS.

308. (Crepidotus) alveolus, Lasch.

309. (Crepidotus) mollis, Scheff.

310. (Crepidotus) Rubi, Berk.

311. (Crepidotus) chimonophilus, B. \& Br.
312. (Crepidotus) variabilis, $P$.

313. (Crepidotus) depluens, Batsch.

314. (Crepidotus) byssisedus, $P$.

315. (Crepidotus) Pezizoides, Nees.

D.-Pratelle.

Sub-Genus 23.-PSALLTOTA.

316. (Psalliota) campestris, $L$.

317. (Psalliota) arvensis, Schaff.

318. (Psalliota) cretaceus, Fr.

319. (Psalliota) silvaticus, Schaffr.

320. (Psalliota) echinatus, Roth.

321. (Psalliota) Jerdoni, Berk.

322. (Psalliota) æruginosus, Curt.

323. (Psalliota) albocyaneus, Desm

324. (Psalliota) melaspermus, Bull.

325. (Psalliota) squamosus, $\mathrm{Fr}$.

326. (Psalliota) stercorarius, $\mathrm{Fr}$.

327. (Psalliota) semiglobatus, Batsch.

Sub-Genus 24.-HYPHOLOMA.

328. (Hypholoma) sublateritius,

329. (Hypholoma) capnoides, $F r$.

330. (Hypholoma) epixanthus, Fr.

331. (Hypholoma) fascicularis, IIuds.

332. (Hypholoma) dispersus, Fr.

333. (Hypholoma)lacrymabundus, Fr.

334. (Hypholoma) velutinus, $P$.

335. (Hypholoma) appendiculatus, Bull.

336. (Hypholoma) egenulus, Berk. 337. (Hypholoma) Candollianus, Fr.

338. (Hypholoma) lanaripes, Cooke. 
Sub-Genus 25.-PSILOCYBE. 339. (Psilocybe) spadiceus, Schaff. 340. (Psilocybe) cernuus, Müll. 341. (Psilocybe) clivensis, Berk. 342. (Psilocybe) Fœnisecii, $P$. 343. (Psilocybe) coprophilus, Bull. 344. (Psilocybe) bullaceus, Bull. 345. (Psilocybe) physaloides, Bull. 346. (Psilocybe) areolatus, Klotsch. 347. (Psilocybe) semilanceolatus, Fr.

348. (Psilocybe) comptulus, Berk.

Sub-Genus 26.-PSATHYRA. 349. (Psathyra) conopilus, $P$. 350. (Psathyra) mastiger, Berk. 351. (Psathyra) corrugis, $P$. 352. (Psathyra) bifrons, Berk. 353. (Psathyra) spadiceo-griseus, Schaff.

354. (Psathyra) cbtusatus, Fr. 355. (Psathyra) fibrillosus, $P$. 356. (Psathyra) urticæcola, Berk. 357. (Psathyra) gossypinus, Fr. 358. (Psathyra) semivestitus, Berk. 359. (Psathyra) Gordoni, Berk.

\section{2.-COPRINARII.}

Sub-Genus 27.-PAN EOLUS. 360. (Panæolus) separatus, $L$. 361. (Panæolus) fimiputris, Bull. 362. (Panæolus) Phalænarum, Fr. 363. (Panæolus) retirugis, Batsch. 364. (Panæolus) campanulatus, $L$. 365. (Panæolus) papilionaceus, Bull.

366. (Panæolus) sub-balteatus,
367. (Panæolus) flmicola, Fr.

Sub-Genus 28.-PSATHYRELLA.

368. (Psathyrella) gracilis, $F r$.

369. (Psathyrella) hiascens, $F r$.

370. (Psathyrella) aratus, Berk.

371. (Psathyrella) pronus, Fr.

372. (Psathyrella) atomatus, Fr.

373. (Psathyrella) disseminatus, $P$.

GENUS II.-COPRINUS, Fr.

374. Coprinus comatus, Fr.

375. Coprinus ovatus, Fr.

376. Coprinus sterquilinus, Fr. 377. Coprinus atramentarius, $F r$. 378. Coprinus fuscescens, Fr. 379. Coprinus picaceus, Fr. 380. Coprinus aphthosus, Fr. 381. Coprinus flocculosus, Fr. 382. Coprinus extinctorius, Fr. 383. Coprinus fimetarius, $F r$. 384. Coprinus tomentosus, Fr. 385. Coprinus niveus, Fr. 386. Coprinus micaceus, $F r$. 387. Coprinus aratus, Berk. 388. Coprinus radians, $F r$. 389. Coprinus deliquescens, $F r$. 390. Coprinus Hendersonii, $F r$. 391. Coprinus macrocephalus, $B$. 392. Coprinus lagopus, Fr. 393. Coprinus nychthemerus, Fr. 394. Coprinus radiatus, $F r$. 395. Coprinus domesticus, $F r$. 396. Coprinus ephemerus, Fr. 397. Coprinus plicatilis, Fr. Berk. 398. Coprinus Spragueii, B. \&C. 
369. Coprinus hemerobius, $F r$. 400. Coprinus filiformis, Berk.

GENUS III.-BOLBITIUS, $F r$. 401. Bolbitius Boltonii, Irr. 402. Bolbitius fragilis, $\mathrm{Fr}$. 403. Bolbitius titubans, Ir. 404. Bolbitius tener, $B$.

GENUS IV.-CORTINARIUS, $F r$. Sub-Genus 1.-PIHEGMACIUM. 405. C. (Phlegmacium) caperatus, 406. C. (Phlegmacium) varius, $F r$. 407. C. (Phlegmacium) cyanopus, Fr.

408. C. (Phlegmacium) anfractus, Fr.

409. C. (Phlegmacium) multiformis, $F r$.

410. C. (Phlegmacium) glaucopus, Fr.

411. C.(Phlegmacium) callochrous, Fr.

412. C. (Phlegmacium) cærulescens, $F r$.

413. C. (Phlegmacium) purpurascens, Fr.

414. C. (Phlegmacium) turbinatus, Fr.

415. C. (Phlegmacium) scaurus, $F r$. 416. C. (Phlegmacium) prasinus, Fr.

Sub-Genus 2.-MYXACIUM.

417. C. (Myxacium) collinitus, $F r$. 418. C. (Myxacium) elatior, $F r$.

419. C. (Myxacium) livido-ochraceus, $B$.

Sub-Genus 3.-INOLOMA.

420. C. (Inoloma) violaceus, $F r$.
421. C. (Inoloma) callistous, $F r$. 422. C. (Inoloma) Bulliardi, Fr. 423. C. (Inoloma) bolaris, $I r$. 424. C. (Inoloma) pholideus, I'r. 425. C. (Inoloma) sublanatus, Iir. 426. C. (Inoloma) arenatus, $P$.

Sub-Genus 4.-DERMOCYBE. 427. C. (Dermocybe) ochroleucus, Fr.

428. C. (Dermocybe) tabularis, $F r$. 429. C. (Dermocybe) diabolicus, $F r$. 430. C. (Dermocybe) caninus, Fr. 431. C. (Dermocybe) anomalus, $F r$. 432. C. (Dermocybo) spilomeus, Fr. 433. C. (Dermocybe) sanguineus, Fr.

434. C. (Dermocybe) cinnamomeus, Fr.

435. C. (Dermocybe) uliginosus, Berk.

436. C. (Dermocybe) raphanoides, Fr.

Sub-Genus 5.-TELAMONIA. 437. C. (Telamonia) bulbosus, Fr. 438. C. (Telamonia) torosus, $F r$. 439. C. (Telamonia) evernius, Fr. 440. C. (Telamonia) armillatus, $F r$. 441. C. (Telamonia) limonius, Fr. 442. C. (Telamonia) hinnuleus, $F r$. 443. C. (Telamonia) Helvelloides, Fr.

444. C. (Telamonia) periscelis, Weinm.

445. C. (Telamonia) psammocephalus, $F r$.

446. C. (Telamonia) ileopodius, $F r$. 447. C. (Telamonia) hemitrichus, Ir. 
Sub-Genus 6.- IYGROCYBE.

44.8. C. (Hygrr ybe) Armeniacus, Fr.

449. C. (Hygrocybe) castaneus, Fr. 450. C. (Hygrocybe) Reedii, Berk. 451. C. (Hygrocybe) rigens, $F r$. 452. C. (Hygrocybe) leucopus, Fr. 453. C. (Hygrocybe) acutus, Fr.

Genus V.-PAXILLUS, Fr. 454. Paxillus involutus, $F r$.

455. Paxillus atrotomentosus, Fr. 456. Paxillus Panuoides, Fr.

GENUS VI.-GOMPHIDIUS, Fr. 457. Gomphidius glutinosus, Fr. 458. Gomphidius viscidus, $F r$. 459. Gomphidius gracilis, $B . \& B r$. GENUS VII. - HYGROPHORUS, Fr.

460. Hygrophorus chrysodon, $F r$. 461. Hygrophorus eburneus, Fr. 462. Hygrophorus cossus, $F r$. 463. Hygrophorus cerasinus, $B$. 464. Hygrophorus mesotephrus, B. \& Br. 465. Hygrophorus arbustivus, $F r$. 466. Hygrophorus hypothejus, Fr. 467. Hygrophorus olivaceo-albus, $F r$.

468. Hygrophorus leporinus, $F r$. 469. Hygrophorus pratensis, $F r$. 470. Hygrophorus virgineus, Fr. 471. Hygrophorus niveus, Fr. 472. Hygrophorus russo-coriaceus, B. \& Mill. 473. Hygrophorus ovinus, $F r$. 474. Hygrophorus distans, $B$.
475. Hygrophorus Colemannianus, Blox.

476. Hygrophorus lætus, Fr.

477. Hygrophorus ceraceus, $\mathrm{Fr}$.

478. Hygrophorus coccineus, Fr. 479. Hygrophorus miniatus, $F r$. 48̀0. Hygrophorus puniceus, $F r$. 481. Hygrophorus obrusseus, Fr. 482. Hygrophorus conicus, Fr. 483. Hygrophorus chlorophanus, Fr.

484. Hygrophorus psittacinus, Fr. 485. Hygrophorus calyptræformis, B. \& Br. 486. Hygrophorus unguinosus, $F r$. 487. Hygrophorus murinaceus, $\mathrm{Fr}$.

GENUS VIII.-LACTARIUS, Fr. 488. Lactarius torminosus, $F r$. 489. Lactarius cilicioides, $F r$. 490. Lactarius turpis, Fr. 491. Lactarius insulsus, $F r$. 492. Lactarius zonarius, $F r$. 493. Lactarius blennius, $\mathrm{Fr}$. 494. Lactarius hysginus, $F r$. 495. Lactarius trivialis, Fr. LID litula 496. Lactarius circellatus, $F r$. 497. Lactarius uvidus, Fr. 498. Lactarius pyrogalus, $F r$. 499. Lactarius plumbeus, Fr. 500. Lactarius piperatus, $F r$. 501. Lactarius vellereus, $F r$. 502. Lactarius deliciosus, Fr. 503. Lactarius theiogalus, $F r$. 504. Lactarius chrysorrhœus, Fr. 505. Lactarius acris, $\mathrm{Fr}$. 506. Lactarius pallidus, $F r$. 
507. Lactarius quiotus, Fr.

508. Lactarius volemum, $\mathrm{Fr}$.

508*. Lactarius serifluus, Fr.

509. Lactarius mitissimus, $F r$.

510. Lactarius subdulcis, $F r$.

511. Lactarius camphoratus, $F r$.

512. Lactarius rufus, $F r$.

513. Lactarius glyciosmus, Fr.

514. Lactarius fuliginosus, $F r$.

GENUS IX.-RUSSULA, Fr.

515. Russula nigricans, Fr.

516. Russula adusta, Fr.

517. Russula delica, $F r$.

518. Russula sanguinea, Fr.

519. Russula rosacea, $F r$.

520. Russula furcata, Fr.

521. Russula vesca, Fr.

522. Russula heterophylla, Fr.

523. Russula depallens, $F r$.

524. Russula sardonia, Fr.

525. Russula rubra, Fr.

526. Russula lepida, Fr.

527. Russula virescens, Fr.

528. Russula emetica, Fr.

529. Russula ochroleuca, Fr.

530. Russula fœtens, $F r$.

531. Russula fragilis, $F r$.

532. Russula integra, Fr

533. Russula aurata, Fr.

534. Russula decolorans, Fr.

535. Russula nitida, Fr.

536. Russula alutacea, Fr.

537. Russula lutea, Fr.

538. Russula vitellina, $\mathrm{Fr}$.
GENUS X.-CANTHARELLUS, Fr.

539. Cantharellus cibarius, $F r$.

540. Cantharellus aurantiacus, $F r$.

541. Cantharellus Brownii, B. \& Br.

542. Cantharellus umbonatus, $P$.

543. Cantharellus tubæformis, Fr.

514. Cantharellus infundibuliformis, $F r$.

515. Cantharellus muscigenus, $F r$.

546. Cantharellus retirugus, $F r$.

547. Cantharellus lobatus, $F r$.

GENUS XI.-NYCTALIS, $F r$.

548. Nyctalis asterophora, Fr.

549. Nyctalis parasitica, Fr.

GENUS XII.-MARASMIUS, Fr.

550. Marasmius urens, Fr.

551. Marasmius peronatus, $F r$.

552. Marasmius porreus, $F r$.

553. Marasmius oreades, $F r_{\bullet !}$

554. Marasmius fusco-purpureus,

Fr.

555. Marasmius Wynnei, $B . \& B r$.

556. Marasmius Stephensii, B.\&Br.

557. Marasmius erythropus, $F r$.

558. Marasmius archyropus, Fr.

559. Marasmius scorodonius, $F r$.

560. Marasmius Vaillantii, $F r$.

561. Marasmius fœtidus, $F r$.

562. Marasmius nigricans, Berk.

MSS.

563. Marasmius amadelphus, Fr.

564. Marasmius ramealis, $F r$.

565. Marasmius androsaceus, Fr.

566. Marasmius rotula, $F r$.

567. Marasmius graminum, B.\&Br 
568. Marasmius alliaceus, Fr.

569. Marasmius perforans, Fr.

570. Marasmius insititius, Fr.

571. Marasmius Hudsoni, Fr.

572. Marasmius saccharinus, Fr.

573. Marasmius epiphyllus, Fr.

574. Marasmius spodoleucus, $B$.

$\& B r$

GENUS XIII.-LENTINUS, $F r$.

575. Lentinus tigrinus, Fr.

576. Lentinus Dunalii, Fr.

577. Lentinus lepideus, Fr.

578. Lentinus cochleatus, Fr.

579. Lentinus vulpinus, Fr.

GENUS XIV.-PANUS, Fr.

580. Panus torulosus, Fr.

j81. Panus conchatus, Fr.

i82. Panus stypticus, Fr.

GENUS XV.-XEROTUS, Fr.

¡83. Xerotus degener, Fr.

GENUS XVI.- SCHIZOPHYLLUM, Fr.

584. Schizophyllum commune, $F r$. GENUS XVII.-LENZITES, Fr.

585. Lenzites betulina, Fr.

586. Lenzites flaccida, $\mathrm{Fr}$.

587. Lenzites sepiaria, Fr.

588. Lenzites abietina, Fr.
DOUBTFUL OR EXTINCT SPECIES.

\section{AGARICUS.}

(Tricholoma) albellus, D.C.

(Clitocybe) vernicosus, $\mathrm{Fr}$.

(Collybia)? œdematopus, Schaff.

(Mycena) tersllus, Schum.

(IMycena) cruentus, Fr.

(Mycena) chelidonius, Fr.

(Omphalia) Oniscus, $F r$.

(Entoloma) ardosiacus, Bull.

(Pholiota) comosus, Fr.

(Flammula) inopus, $F r$.

(Naucoria) nuceus, Bolt.

(Galera)? pilipes, Sow.

(Crepidotus) haustellaris, Fr.

(Psalliota) versicolor, With.

(Panæolus) cinctulus, Bolt.

Coprinus luridus, Fr.

Cortinarius (Tel.) brunneus, Fr.

Cortinarius (Hyg.) dilutus, $F r$.

Hygrophorus aromaticus, Berk.

Cantharellus cinereus, $F r$.

Marasmius candidus, $F r$.

Lentinus adhærens, Fr.

Lentinus flabelliformis, $F r$.

ADDENDA.

8* (Amanita) spissus, Fr. 579* Lentinus fimbriatus, Curr. 


\section{ORDER II.-POLYPOREI.}

GENUS I.-BOLETUS, Fr.

589. Boletus luteus, $L$.

590. Boletus elegans, Schum.

591. Boletus flavus, With.

592. Boletus laricinus, $B$.

593. Boletus granulatus, $L$.

594. Boletus bovinus, $L$.

595. Boletus badius, $\mathrm{Fr}$.

596. Boletus sanguineus, With.

597. Boletus piperatus, Bull.

598. Boletus parasiticus, Bull.

599. Boletus variegatus, $F r$.

600. Boletus striæpes, Sec.

601. Boletus chrysenteron, Fr.

602. Boletus subtomentosus, $L$.

603. Boletus calopus, Fr.

604. Boletus olivaceus, Scheff.

605. Boletus pachypus, Fr.

606. Boletus Satanas, Lenz.

607. Boletus luridus, Fr.

608. Boletus erythropus, $P$.

609. Boletus purpureus, Fr.

610. Boletus edulis, Bull.

611. Boletus impolitus, Fr.

612. Boletus æstivalis, Fr.

613. Boletus viscidus, $L$.

614. Boletus versipellis, Fr.

615. Boletus scaber, Fr.

616. Boletus alutarius, Frr.

617. Boletus felleus, Bül..
618. Boletus castaneus, Bull.

GENUS II.-STROBILOMYCES, $B$.

619. Strobilomyces strobiliaceus, $B$.

GENUS III.-POLYPORUS, Fr.

620. Polyporus brumalis, $F r$.

621. Polyporus fuscidulus, $F r$.

622. Polyporus leptocephalus, $F r$.

623. Polyporus lentus, Berk.

624. Polyporus Schweinitzii, $\mathrm{Fr}$

625. Polyporus rufescens, $F r$.

626. Polyporus perennis, $F r$.

627. Polyporus squamosus, $F r$.

628. Polyporus Rostkovii, Fr.

629. Polyporus picipes, $F r$.

630. Polyporus varius, $F r$.

631. Polyporus elegans, Fr.

632. Polyporus quercinus, Fr.

633. Polyporus lucidus, Fr.

634. Polyporus intybaceus, $F r$.

635. Polyporus cristatus, Fr.

636. Polyporus giganteus, $F r$.

637. Polyporus sulfureus, $F r$.

638. Polyporus alligatus, Fr.

639. Polyporus heteroclitus, Fr.

640. Polyporus salignus, Fr.

641. Polyporus chioneus, $F r$.

642. Polyporus fragilis, Frs.

643. Polyporus cæsius, $F r$. 
644. Polyporus destructor, Fr.

645. Polyporus nidulans, Fr.

646. Polyporus rutilans, $F r$.

647. Polyporus fumosus, $F r$.

648. Polyporus adustus, Fr.

649. Polyporus crispus, $F r$.

650. Polyporus adiposus, $B . \& B r$.

651. Polyporus amorphus, $F r$.

652. Polyporus hispidus, $\mathrm{Fr}$.

653. Polyporus spumeus, $F r$.

654. Polyporus dryadeus, Fr.

655. Polyporus betulinus, Fr.

656. Polyporus pallescens, Fr.

657. Polyporus vegetus, $F r$.

658. Polyporus applanatus, $F r$.

659. Polyporus fomentarius, $F r$.

660. Polyporus nigricans, Fr.

661. Polyporus igniarius, Fr.

662. Polyporus ribis, Fr.

663. Polyporus conchatus, $F r$.

664. Polyporus salicinus, Fr.

665. Polyporus ulmarius, Fr.

666. Polyporus fraxineus, $F r$.

667. Polyporus cytisinus, Berk.

668. Polyporus variegatus, $F r$.

669. Polyporus cervinus, $P$.

670. Polyporus annosus, $F r$.

671. Polyporus connatus, Fr.

672. Polyporus radiatus, Fr.

673. Polyporus velutinus, $F r$.

674. Polyporus versicolor, $F r$.

675. Polyporus abietinus, Fr.

76. Polyporus Wynnei, $B . \& B r$.

677. Polyporus contiguus, $F r$.

678. Polyporus ferruginosus, $F r$.
679. Poly porus nitidus, Fr.

680. Polyporus Armeniacus, Berk.

681. Polyporus bombycinus, $F r$.

682. Polyporus incarnatus, $F r$.

683. Polyporus purpureus, $\mathrm{Fr}$.

684. Polyporus cinctus, Berk.

685. Polyporus medulla-panis, Fr.

686. Polyporus vitreus, Fr.

687. Polyporus obducens, Fr.

688. Polyporus vulgaris, $F r$.

689. Polyporus viridans, Berk.

690. Polyporus molluscus, Fr.

691. Polyporus tęrrestris, Fr.

692. Polyporus vaporarius, $F r$.

693. Polyporus aneirinus, Fr.

694. Polyporus Stephensii, Fr.

695. Polyporus Vaillantii, Fr.

696. Polyporus hybridus, $B$. \& Br.

GENUS IV.-TRAMETES, Fr.

697. Trametes pini, $\mathrm{Fr}$.

698. Trametes suaveolens, Fr.

699. Trametes odora, Fr.

700. Trametes gibbosa, $F r$.

GENUS $V$.-D正DALEA, $F r$.

701. Dædalea quercina, $P$.

702. Dædalea confragosa, $\boldsymbol{P}$.

703. Dædalea unicolor, $\mathrm{Fr}$.

704. Dædalea latissima, $F r$.

GENUS VI.-MERULIUS, Fr.

705. Merulius tremellosus, Schrad.

706. Merulius corium, Fr.

707. Merulius molluscus, $F r$.

708. Merulius Porinoides, Fr.

709. Merulius rufus, $P$. 
710. Merulius serpens, $I r$.

711. Merulius pallons, Berk.

712. Merulius Carmichaelianus, Berk.

713. Merulius lacrymans, $F^{r} r$.

714. Merulius aurantiacus, Klotsch.

GENUS VII.-POROTHELIUM, Fr.

715. Porothelium Friesii, Mont.

\section{GENUS VIII.-FISTULINA,} Bull.

716. Fistulina hepatica, Ifr

DOUBTFUL OR EXTINCT SPECIES.

Boletus cyanescens, Bull.

Polyporus fibula, Fr.

\section{ORDER III.-HYDNEI.}

GENUS I.-HYDNUIM, $L$.

717. Hydnum imbricatum, $L$.

718. Hydnum repandum, $L$.

719. Hydnum compactum, Fr.

720. Hydnum zonatum, Batsch.

721. Hydnum graveolens, Del.

722. Hydnum auriscalpium, $L$.

723. Hydnum coralloides, Scop.

724. Hydnum Erinaceus, Bull.

725. Hydnum Caput-Medusæ, Bull

726. Hydnum gelatinosum, Scop.

727. Hydnum ochraceum, $P$.

728. Hydnum membranaceum, Bull.

729. Hydnum Weinmanni, Fr.

730. Hydnum fuscum, $P$.

731. Hydnum ferruginosum, Fr.

732. Hydnum variecolor, $P$.

733. Hydnum alutaceum, Fr.

734. Hydnum spathulatum, Fr.

735. Hydnum udum, Fr.
736. Hydnum niveum, $\boldsymbol{P}$.

737. Hydnum farinaceum, $P$.

738. Hydnum plumosum, Duby.

GENUS II.-SISTOTREMA, Fr.

739. Sistotrema confluens, $P$.

GENUS III.-IRPEX, $F r$.

740. Irpex pendulus, $F r$.

741. Irpex Johnstoni, Berk.

742. Irpex obliquus, $F r$.

GENUS IV.-Radulum, $F r$.

743. Radulum orbiculare, Fr.

744. Radulum quercinum, $F r$.

GENUS V.-PHLEBIA, Fr.

745. Phlebia merismoides, Fr.

746. Phlebia radiata, Fr.

747. Phlebia contorta, Fr.

748. Phlebia vaga, $F r$.

GENUS VI.-GRANDINIA, $F r$.

749. Grandinia granulosa, $F r$. 
GENUS VII.-ODONTIA, Fr.

750. Odontia fimbriata, $\mathrm{Fr}$.

GENUS VIII.-KNEIFFIA, $F r$.

751. Kneiffia setígera, Fr.
DOUB'TFUL OR EX'TINCT SPECIES.

Hydnum squalinum, $\mathrm{Fr}$.

Hydnum crispum, Schaff.

\section{ORDER IV.-AURICULARINI.}

GENUS I.-CRATERELLUS, $F r$.

752. Craterellus Iutescens, Fr.

753. Craterellus cornucopioides,

754. Craterellus sinuosus, $F r$.

755. Craterellus crispus, Fr.

GENUS II.-THELEPHORA, Fr.

756. Thelephora Sowerbeii, Berk.

757. Thelephora tuberosa, Grev.

758. Thelephora anthocephala, Fr.

759. Thelephora caryophyllæa, Fr.

760. Thelephora palmata, Fr.

761. Thelephora terrestris, Fr.

762. Thelephora cristata, Fr.

763. Thelephora fastidiosa, $F r$.

764. Thelephora mollissima, $P$.

765. Thelephora laciniata, $P$.

766. Thelephora biennis, Fr.

767. Thelephora cæsia, $P$.

768. Thelephora byssoides, $P$.

769. Thelephora sebacea, Fr.

770. Thelephora puteana, Schum.

771. Thelephora laxa, Fr.

772. Thelephora arida, $F r$.
773. Thelephora olivacea, $F r$.

774. Thelephora anthochroa, $P$.

GENUS III.-STEREUM, Fr. 775. Stereum purpureum, Fr.

776. Stereum hirsutum, $F r$.

777. Stereum spadiceum, $\mathrm{Fr}$.

778. Stereum sanguinolentum, Fr.

779. Stereum rugosum, $F r$.

750. Stereum acerinum, $F r$.

GENUS IV.-HYMENOCH画TE, Lév.

781. Hymenochæto rubiginosa, Lév.

782. Hymenochæte tabacina, Lév.

783. Hymenochæte corrugata, Berk.

GENUS $V$.-AURICULARIA, Fr. 784. Auricularia mesenterica, Bull. 785. Auricularia lobata, Somm.

GENUS VI.-CORTICIUM, $F r$. 786. Corticium giganteum, $\mathrm{Fr}$. 787. Corticium lacteum, $\mathrm{Fr}$. 788. Corticium arachnoideum,Berk 789. Corticium læve, Fr. 
790. Corticium roseum, $P$.

791. Corticium velutinum, $F r$.

792. Corticium sanguineum, $\mathrm{Fr}$.

793. Corticium sulfuroum, $F r$.

794. Corticium cœruleum, Fr.

795. Corticium atrovirens, $F r$.

796. Corticium lactescens, Bert.

797. Corticium calceum, $F r$.

798. Corticium lividum, $P$.

799. Corticium ochraceum, Fr.

800. Corticium quercinum, $P$.

801. Corticium cinereum, $F r$.

802. Corticium incarnatum, $F r$.

803. Corticium nudum, Fr.

804. Corticium confluens, Fr.

805. Corticium polygonium, $P$.

806. Corticium comedens, Fr.

807. Corticium Sambuci, $P$.

808. Corticium Aurora, B. \& Br.
GENUS VII.-CYPHELLA, Fr. 809. Cyphella griseopallida, $F r$.

810. Cyphella muscigena, $\mathrm{Fr}$.

811. Cypholla galeata, Fr.

812. Cyphella ochroleuca, $B . \& B r$.

813. Cyphella muscicola, Fr.

814. Cyphella lacera, $\mathrm{Fr}$.

815. Cyphella capula, Fr.

816. Cyphella Goldbachii, Fr.

817. Cyphella Curreyi, Berk.

818. Cyphella fulva, B. \& Rav.

\section{DOUBTFUL OR EXTINCT}

SPECIES.

Thelephora cretacea, $P$.

Thelephora dryina, $P$.

Cyphella cuticulosa, Fr.

\section{Order V.-CLAVARIEI.}

GENUS I.-CLAVARIA, $L$. 819. Clavaria Botrytis, $P$. 820. Clavaria amethystina, Bull. 821. Clavaria fastigiata, D. C. 822. Clavaria muscoides, $L$. 823. Clavaria coralloides, $L$. 824. Clavaria umbrina, Berk. 825. Clavaria cinerea, Bull. 826. Clavaria cristata, Holmsk.
827. Clayaria rugosa, Bull. 828. Clavaria Kunzei, Fr. 829. Clavaria aurea, Schaff. 830. Clavaria abietina, Schum. 831. Clavaria flaccida, $F r$. 832. Clavaria crocea, $P$. 833. Clavaria grisea, $P$. 834. Clavaria stricta, $P$. 835. Clavaria crispula, $F r$. 
836. Clavaria purpurea, Mrüll.

837. Clavaria rosea, $F r$.

838. Clavaria fusiformis, Sow.

839. Clavaria ceranoides, $P$.

840. Clavaria inæqualis, Müll.

841. Clavaria argillacea, $F r$.

842. Clavaria tenuipes, $B . \& B r$.

843. Clavaria vermiculata, Scop.

844. Clavaria fragilis, Holmsk.

845. Clavaria pistillaris, $L$.

846. Clavaria contorta, $F r$.

847. Clavaria Ardenia, Sow.

848. Clavaria juncea, Fr.

849. Clavaria acuta, Sow.

850. Clavaria uncialis, Grev.

GENUS II.-CALOCERA, Fr.

851. Calocera viscosa, $\mathrm{Fr}$.

852. Calocera cornea, Fr.

853. Calocera glossoides, Fr.
GENUS 1II.-TYPHULA, Fr. 854. Typhula erythropus, Fr.

855. Typhula phacorrhiza, Fr.

856. Typhula incarnata, Fr.

857. Typhula muscicola, Fr.

858. Typhula Grevillei, $F r$.

859. Typhula filiformis, $F r$.

860. Typhula gracilis, B. \& Desm.

GENUS IV.-PISTILLARIA, Fr.

861. Pistillaria micans, $F r$.

862. Pistillaria culmigena, Mont.

863. Pistillaria quisquilaris, $F r$.

864. Pistillaria puberula, Berk.

865. Pistillaria pusilla, Fr.

DOUBTFUL OR EXTINCT SPECIES.

Calocera tuberosa, $F r$.

\section{ORDER VI.-TREMELLINI.}

GENUS I.-TREMELLA, Fr. 866. Tremella frondosa, $F r$. 867. Tremella foliacea, $P$. 868. Tremella lutescens, Fr. 869. Tremella mesenterica, Retz. 870. Tremella moriformis, Berk. 871. Tremella albida, Huds. 872. Tremella intumescens, Sow. 873. Tremella indecorata, Somm.
874. Tremella sarcoides, $S m$. 875. Tremella clavata, $P$.

876. Tremella tubercularia, Bull. 877. Tremella torta, Willd. 878. Tremella versicolor, $B . \& B r$. 879. Tremella viscosa, $P$. 880. Tremella epigæa, $B . \& B r$. GENUS II.-EXIDIA, Fr. 881. Exidia recisa, $F r$. 
882. Exidia glandulosa, $F r$.

883. Exidia saccharina, $\mathrm{Fr}$.

GENUS III.-HIRNEOLA, Fr.

884. Hirneola auricula-Judæ, Berk.

$G E N U S I V$-N \#MATELIA, $F r$.

885. Næmatelia encephala, $F r$.

886. Næmatelia nucleata, Fr.

887. Næmatelia virescens, $C d$.

GENUS V.-DACRYMYCES, Nees.

888. Dacrymyces violaceus, Fr.

889. Dacrymyces deliquescens, Duby.

890. Dacrymyces stillatus, Nees.
891. Dacrymyces chrysocomus, Tul. GENUS VI.-APYRENIUM, Fr. 892. Apyrenium lignatile, Fr. GENUS VII.-HYMENULA, Fr. 893. Hymenula punctiformis,

B. \& Br.

GENUS VIII.-DITIOLA, Fr. 894. Ditiola radicata, $F r$.

DOUBTFUL OR EX'TINCT SPECIES.

Tremella fimbriata, $P$.

Tremella vesicaria, Bull. 


\section{FAMILY II.-GASTEROMYCETES.}

\section{ORDER VII.-HYPOGAI.}

GENUS I._OCTAVIANIA, Vitt.

895. Octaviania asterosperma, Vitt.

896. Octaviania Stephensii, Tul.

GENUS II.-MELANOGASTER, $C d$.

897. Melanogaster variegatus, Tul.

897A. Melanogaster variegatus, var. Broomeianus, Berk.

898. Melanogaster ambiguus, Tul.

898A. Melanogaster ambiguus, var. $\beta$, intermedius, Berk.

\section{GENUS III.-HYDNANGIUM, Wallr.}

899. Hydnangium carotæcolor, Berk.

\section{GENUS IV.-HYSTERANGIUM,} Vitt.

900. Hysterangium nephriticum,

901. Hysterangium Thwaitesii, Berk. B. $\& B r$.
GENUS V.-RHIZOPOGON, Tul.

902. Rhizopogon rubescens, Tul. GENUS VI.-HYMENOGASTER, Tul.

903. Hymenogaster Klotschii, Tul. 904. Hymenogaster muticus, B. \& $B r$. 905. Hymenogaster luteus, Vitt. 906. Hymenogaster decorus, Tul. 907. Hymenogaster vulgaris, Tul. 908. Hymenogaster pallidus, B. \&Br. 909. Hymenogaster citrinus, Vitt. 910. Hymenogaster olivaceus, Vitt. 911. Hymenogaster tener, Berk. 912. Hymenogaster Thwaitesii, B. \&Br. 913. Hymenogaster pusillus, B. \&Br.

\section{ORDER VIII.-PHALLOIDEI.}

GENUS I.-PHALLUS, $L$. 914. Phallus impudicus, $L$. 915. Phallus iosmos, Berk.

\section{GENUS II.-CYNOPHALLUS,} Fr.

916. Cynophallus caninus, $F r$. GENUS III.-CLATHRUS, Mich. 917. Clathrus cancellatus, $L$. 


\section{Order IX.-TRICHOGASTRES.}

GENUS I.-BATARREA, $P$. 918. Batarrea phalloides, $P$.

GENUS II.-TULOSTOMA, $P$. 919. Tulostoma mammosum, $\mathrm{Fr}$.

GENUS III.-GEASTER, Mich. 920. Geaster coliformis, $P$.

921. Geaster fornicatus, $F r$.

922. Geaster striatus, D.C.

923. Geaster Bryantii, Berk.

924. Geaster limbatus, $F r$.

925. Geaster fimbriatus, $F r$.

926. Geaster rufescens, $P$.

927. Greaster hygrometricus, $P$.

GENUS IV.-BOVISTA, Dill.

928. Bovista nigrescens, $P$.

929. Bovista plumbea, $P$.
GENUS $V$.-IYCOPERDON, Tourn.

930. Lycoperdon giganteum,
931. Lycoperdon cælatum, Fr.

932. Lycoperdon atropurpureum,

Vitt.

933. Lycoperdon pusillum, Fr.

934. Lycoperdon saccatum, Vahl.

935. Lycoperdon gemmatum, $\mathrm{Fr}$.

936. Lycoperdon pyriforme, Schaeff.

\section{GENUS VI.-SCLERODERMA, $P$.}

937. Scleroderma vulgare, $F r$.

938. Scleroderma Bovista, Fr.

939. Scleroderma verrucosum,Pers.

GENUS VII.-CENOCOCCUM, Fr.

940. Cenococcum geophilum, Fr.

\section{DOUBTFUL OR EXTINCT} SPECIES.

Geaster mammosus, Chev. Polysaccum olivaceum, Fr.

\section{ORDER X.-MYXOGASTRES.}

GENUS I. - IYCOGALA, IIich.

941. Lycogala epidendrum, $F r$. 942. Lycogala parietinum, $F r$.

\section{GENUS II.-RETICULARIA,} Bull.

943. Reticularia maxima, $F r$.

944. Reticularia atra, Fr.

945. Reticularia umbrina, $F r$. 
GENUS III.-\#THALIUM, LK.

946. 怍thalium septicum, Fr.

947. 开thalium vaporarium, Fr.

GENUS IV.-SPUMARIA, $P$.

948. Spumaria alba, $D C$.

GENUS $V$.-DIDERMA, $P$. 919. Diderma floriforme, $P$.

950. Diderma umbilicatum, $P$.

951. Diderma citrinum, Fr.

952. Diderma vernicosum, $P$.

953. Diderma lucidum, Berk.

954. Diderma spumarioides, $F r$.

955. Diderma Trevelyani, Fr.

956. Diderma Carmichaelianum, Berk.

957. Diderma nitens, Klotsch.

958. Diderma globosum, $F r$.

959. Diderma cyanescens, Fr.

960. Diderma deplanatum, Fr.

961. Diderma contextum, $P$.

GENUS VI.-DIDYIMIUM, Schrad.

962. Didymium melanopus, Fr.

962a. Didymium melanopus, var. $\beta$ clavus.

963. Didymium hemisphæricum, Fr.

964. Didymium furfuraceum, Fr.

965. Didymium tigrinum, Fr.

966. Didymium squamulosum, A. \& S.

967. Didymium farinaceum, $F r$.

968. Didymium nigripes, $F r$.

969. Didymium pertusum, Berk.

970. Didymium xanthopus, $F r$.

971. Didymium leucopus, $F r$.

972. Didymium Sowerbeii, Ber/.
973. Didymium lobatum, Nees.

974. Didymium congestum, B. \& $\mathrm{Br}$.

975. Didymium dædaleum,

B. \& $B r$.

976. Didymium physaroides, $F r$.

977. Didymium cinereum, $F r$.

978. Didymium serpula, $F r$.

GENUS VII.-PHYSARUM, $P$. 979. Physarum nutans, $P$.

979^. Physarum nutans, var. $\gamma$ aureum, Grev.

980. Physarum bulbiforme, Schum.

981. Physarum rubiginosum, Chev.

982. Physarum lilacinum, $F r$.

983. Physarum metallicum, Berk.

984. Physarum album, Fr.

985. Physarum atrum, Fr. GENUS VIII.-ANGIORIDIUM,
Grev.

986. Angioridium sinuosum, Grev. GENUS IX.-BADHAIMIA, Berk.

987. Badhamia hyalina, Berk.

988. Badhamia utricularis, Berk.

989. Badhamia nitens, Berk.

990. Badhamia pallida, Berk.

991. Badhamia fulvella, Berk.

GENUS X.-CRATERIUM, Trent. 992. Craterium pedunculatum,

Trent.

993. Craterium pyriforme, Ditm.

994. Craterium minutum, Fr.

995. Craterium leucocephalum, Ditm

996. Craterium mutabile, $F r$.

GENUS XI.-DIACHEA, Fr. 997. Diachea elegans, Fr. 


\section{GENUS XII.-STEMONITIS, Gled.}

998. Stemonitis fusca, Roth. 999. Stemonitis ferruginea, Ehib. 1000. Stemonitis typhoides, $D C$. 1001. Stomonitis ovata, $P$.

1002. Stemonitis obtusata, $\mathrm{Fr}$. 1003. Stemonitis pulchella, Bab. 1004. Stemonitis physaroides,

1005. Stemonitis violacea, $F r$. 1006. Stemonitis arcyrioides, Somm.

\section{GENUS XIII.-ENIRTHE- NEMA, Bowm.}

1007. Enerthenema elegans, Bowm.

\section{GENUS XIV.-DICTYDIUM, Schrad.}

1008. Dictydium umbilicatum, Schrad.

\section{GENUS $X V$.-CRIBRARIA,} Schrad.

1009. Cribraria intermedia, Berk. 1010. Cribraria intricata, Schrad.

GENUS XVI.-ARCYRIA, Hill. 1011. Arcyria punicea, $P$. 1012. Arcyria incarnata, $P$ 1013. Arcyria cinerea, Schum. 1014. Arcyria nutans, Fr. 1015. Arcyria umbrina, Schum. 1016. Arcyria ochroleuca, Fr.
GENUS XVII.-OPHIOTHECA, Curr.

1017. Ophiotheca chrysosperma, Curr GENUS XVIII.-TRICHIA, Hall 1018. Trichia rubiformis, $P$. 1019. Trichia pyriformis, Hoffm. 1020. Trichia Ayresii, B. \& Br. 1021. Trichia Lorinseriana, $\mathrm{Cl}$. 1022. Trichia serotina, Schrad. 1023. Trichia fallax, $P$. 1024. Trichia clavata, $P$. 1025. Trichia cerina, Ditm. 1026. Trichia nimripes, $P$. 1027. Trichia turbinata, With. 1028. Trichia chrysosperma, $D C$. 1029. Trichia varia, $P$. 1030. Trichia serpula, $P$.

\section{GENUS XIX.-PERICHÆNA, Fr.}

1031. Perichæna abietina, Fr.

1032. Perichæna populina, Fr.

GENUS XX.-LICEA, Schrad. 1033. Licea cylindrica, $F r$. 1034. Licea fragiformis, $F r$. 1035. Licea applanata, Berk. 1036. Licea perreptans, Berk GENUS XXI.- PHELONITIS, Chev.

1037. Phelonitis strobilna, Fr. 


\section{Order XI.-NIDULARIACEI.}

GENUS I.-CYATHUS, $P$. 1038. Cyathus striatus, Hoffm. 1039. Cyathus vernicosus, $D C$.

GENUS II.-CRUCIBULUM, Tul. 1040. Crucibulum vulgare, Tul.

GENUS III.-SPH \#ROBOIUS, Tode.

1041. Sphærobolus stellatus, Tode.

GENUS III.*-NIDULARIA, Bull.

1041* Nidularia pisiformis, Tul.
GENUS IV.-POLYANGIUM, Lk.

1042. Polyangium vitellinum, Ditm.

DOUBTFUL OR EXTINCT SPECIES.

Myriococcum præcox, $F r$.

Thelebolus stercoreus, Tode.

\section{A D D E N A.}

991* Badhamia inaurata, Curr. 


\section{FAMILY III.CCONIOMYCETES.}

\section{ORdER XII.-SPIIARONEMEI.}

\section{GENUS I.-CONIOTHYRIUM,} $C d$.

1043. Coniothyrium glomeratum,

GENUS II.--LEPTOSTROMA, Fr.

1044. Leptostroma caricinum, $F r$. 1015. Leptostroma juncinum, Fr. 1046. Leptostroma filicinum, $F r$. 1047. Leptostroma litigiosum, Desm.

1048. Leptostroma spireæ, Fr. 1049. Leptostroma vulgare, Fr. GENUS III.-PHOMA, Fr. 1050. Phoma concentricum, Desm. 1051. Phoma hederæ, Desm. 1052. Phoma asteriscus, Berk. 1053. Phoma nothum, Berk. 1054. Phoma lingam, Desm. 1055. Phoma radula, $B . \& B r$. 1056. Phoma depressum, B. \&Br. 1057. Phoma eriophorum, B. \&Br. 1058. Phoma samarorum, Desm. 1059. Phoma piceum, B. \&Br . 1060. Phoma sticticum, $B . \& B r$. 1061. Phoma exiguum, Desm. 1062. Phoma devastatrix, B. \& Br. 1063. Phoma microscopicum, $B$. $\& B r$.

1061. Phoma nebulosum, Berk.
1065. Phoma longissimum, Berk. 1066. Phoma inophilum, Berk. 1067. Phoma muciferum, Berk. 1068. Phoma ulmicola, Berk. 1069. Phoma epileucum, Berk. 1070. Phoma fibricola, Berk. 1071. Phoma bicuspidatum, Berk. GENUS IV.-LEPTOTHYRIUM, Kze.

1072. Leptothyrium juglandis, Lib. 1073. Leptothyrium fragariæ, Lib. 1074. Leptothyrium ribis, Lib. GENUS $V$.-ACTINOTHYRIUM, Kze.

1075. Actinothyrium graminis, Kze. GENUS VI.-CRYPTOSPORIUII, Kze.

1076. Cryptosporium caricis, $C d$. 1077. Cryptosporium Neesii, $C d$. GENUS VII.-SPHERONEMA, Tode.

1078. Sphæronema subulatum, Tode.

1079. Sphæronema vitreum, $C d$. 1080. Sphæronema leucoconium, B. \& Br.

1081. Sphæronema epimyces, Berk. GENUS VIII.-APOSPH开RIA, Berk.

1082. Aposphæria acuta, Berk. 
1083. Aposphæria complanata, Berk.

GENUS IX.-SPHFROPSIS, Lév. 1084. Sphæropsis atrovirens, Lév. 1085. Sphæropsis Candollii, B.\& Br 1086. Sphæropsis leucostigma, Lév. 1087. Sphæropsis cylindrospora, Desm.

1088. Sphæropsis Ralfsii, $B . \& B r$. 1089. Sphæropsis parca, $B . \& B r$. 1090. Sphæropsis strobi, $B . \& B r$. 1091. Sphæropsis geniculata, $B$. $\& B r$.

1092. Sphæropsis epitricha, $B . \&$ $B r$.

1093. Sphæropsis mutica, $B . \& B r$. 1094. Sphæropsis menispora, $B$. $\& B r$.

1095. Sphæropsis malorum, Berk. 1096. Sphæropsis arundinacea, Lév.

1097. Sphæropsis taxi, Berk. 1098. Sphæropsis alismatis, Curr.

GENUS $X$.-DOTHIORA, Fr. 1099. Dothiora pyrenophora, $F r$. 1100. Dothiora sphæroides, Fr.

GENUS XI.-CLINTERIUM, Fr. 1101. Clinterium obturatum, Fr.

GENUS XII.-ACROSPERMUM, Tode.

1102. Acrospermum compressum, Tode.

1103. Acrospermum graminum, Lib.

GENUS XIII.-DIPLODIA, Fr.

1104. Diplodia mutila, Fr.

1105. Diplodia confluens, B. \&Br.
1106. Diplodia cæspitosa, $B . \& B r$. 1107. Diplodia vulgaris, Lév. 1108. Diplodia ilicicola, Desm. 1109. Diplodia viticola, Desm. 1110. Diplodia paupercula, B.\&Br. 1111. Diplodia Cowdellii, B. \& Br. 1112. Diplodia fibricola, Berk. 1113. Diplodia oospora, Berk. 1114. Diplodia tecta, $B . \& B r$. 1115. Diplodia consors, $B . \& B r$. 1116. Diplodia arbuticola, Fr. 1117. Diplodia ilicis, Curr.

GENUS $X I V$ - HENDERSONIA, Berk.

1118. Hendersonia elegans, Berk.

1119. Hendersonia macrospora, $B$. $\& B r$.

1120. Hendersonia arcus, $B . \& B r$. 1121. Hendersonia mutabilis, $B . \&$ $B r$.

1122. Hendersonia polycystis, $B$. $\& B r$.

1123. Hendersonia Oreades, Dur. $\&$ Mont.

1124. Hendersonia Stephensii, $B$. $\& B r$.

1125. Hendersonia fibriseda, Berk. GENUS XV.-DARLUCA, Cast. 1126. Darluca filum, Cast.

1127. Darluca typhoidearum, B. \& $B r$. 1128. Darluca macropus, $B . \& B r$. GENUS XVI.-VERMICULARIA Tode.

1129. Vermicularia dematium, $F r$. 1130. Vermicularia trichella, Grev. 1131. Vermicularia atramentaria, B. \& Br. 
1132. Vermicularia circinans, Bert. GENUS XVII.-DISCOSIA, Lib. 1133. Discosia alnea, Lib.

GENUS XVIII.-PILIDIUM, Kze. 1134. Pilidium acerinum, Kze.

1135. Pilidium (?) carbonaceum, Lib.

GENUS XIX.-MELASMIA, Lév.

1136. Melasmia acerina, Lév.

1137. Melasmia alnea, Lév.

GENUS XX.-PIGGOTIA, B. $\oint$ $B r$.

1138. Piggotia astroidea, $B . \& B r$.

GENUS XXI.--SFPTORIA, Fr.

1139. Septoria ulmi, Kze.

1140. Septoria oxyacanthæ, Kze.

1141. Septoria aceris, $B . \& B r$.

1142. Septoria salicella, $B . \& B r$.

1143. Septoria ægopodii, Desm.

1144. Septoria lepidii, Desm.

1145. Septoria nodorum, Berk.

1146. Septoria hippocastani, $B . \&$

1147. Septoria lituus, $B . \& B r$.

1148. Septoria Ralisii, $B . \& B r$.

1149. Septoria insularis, B. \& Br.

1150. Septoria Badhami, B. \& Br.

1151. Septoria polygonorum, Desm.

1152. Septoria convolvuli, Desm.

1153. Septoria cornicola, Desm.

1154. Septoria heterochroa, Desm.

1155. Septoria graminum, Desm.

1156. Septoria stemmatea, Berk.

1157. Septoria hederæ, Desm.
1158. Septoria populi, Desm.

1159. Septoria thecicola, $B . \& B r$. 1160. Septoria princeps, B. \&Br.

\section{GENUS XXII. - ASCOCHYTA,} Lib.

1161. Ascochyta pisi, Lib.

1162. Ascochyta pallor, Berk.

1163. Ascochyta dianthi, Berk.

1164. Ascochyta rufomaculans, Berk.

\section{GENUS XXII.-CYSTOTRI-} CHA, B. \& Br.

1165. Cystotricha striola, $B . \& B r$.

GENUS XXIV.-NEOTTIOSPORIA, Desm.

1166. Neottiosporia caricum, Desm. GENUS $X X V$.-EXCIPULA, $F r$. 1167. Excipula strigosa, $F r$.

1168. Excipula macrotricha, B. \& $B r$.

1169. Fxcipula chætostroma, B. \& $B r$.

1170. Excipula fusispora, $B . \& B r$.

GENUS XXVI.-DINEMASPORIUM, Lév.

1171. Dinemasporium graminum, Lév.

GENUS XXVII.-MYXORMIA, B. $\& B r$.

1172. Myxormia atro-viridis, $B$. $\& B r$.

GENUS XXVIII.-PROSTHEMIUIM, Kze.

1173. Prosthemium betulinum, Kze 1174. Prosthemium stellare, Riess. 


\section{GENUS XXIX.-ASTEROMA,} $D C$.

1175. Asteroma reticulatum, Berk.

1176. Asteroma ulmi, $K l$.

1177. Asteroma prunellæ, Purt.

1178. Asteroma padi, Grev.

1179. Asteroma rosæ, $D C$.

1180. Asteroma veronicæ, Desm.

GENUS XXX.-RABENHORSTIA, Fr.

1181. Rabenhorstia rudis, $F r$.

1182. Rabenhorstia tiliæ, Fr.

GENUS XXXI.-CYTISPORA, Fr.

1183. Cytispora rubescens, $F r$.

1184. Cytispora chrysosperma, Fr.

1185. Cytispora carphosperma, Fr.

1186. Cytispora leucosperma, $P$.

1187. Cytispora Hendersonii, $B . \&$

1188. Cytispora pinastri, Fr.

1189. Cytispora guttifera, Fr.

GENUS XXXII.-MICROPERA, Lév.

1190. Micropera drupacearum, Lév.
GENUS XXXIII.-DISCELLA, B. \& $\mathrm{Br}$.

1191. Discella carbonacea, $B . \& B r$. 1192. Discella Desmazierii, B. \& Br. 1193. Discella platyspora, $B . \& B r$. 1194. Discella microsperma, B. \& $\mathrm{Br}$.

1195. Discella abnormis, $B . \& B r$.

GENUS XXXIV-PHLYCT AENA, Desm.

1196. Phlyctæna vagabunda, Desm.

1197. Phlyctæna Johnstonii, B. \& $B r$.

\section{GENUS XXXV.-CEUTHO- SPORA, $F r$.}

1198. Ceuthospora phacidioides, Grev.

1199. Ceuthospora lauri, Grev.

GENUS XXXVI.-ERIOSPORA, B. \&Br.

1200. Eriospora leucostoma, B. \& $B r$.

\section{ORder XIII.-MELANCONIEI.}

GENUS I.-MELANCONIUM, $L k$.

1201. Melanconium bicolor, Nees. 1202: Melanconium magnum, Berk. 1203. Melanconium sphærospermum, $L k$.
GENUS II.-STEGONOSPO. RIUM, $C d$.

1204. Stegonosporium cellulosum, $C d$.

GENUS III.-STILBOSPORA, $P$. 1205. Stilbospora ovata, $P$.

1206. Stilbospora angustata, $P$. 
GENUSIV.-ASTEROSPORIUM, Iize.

1207. Asterosporium Hoffmanni, M. \& $N$.

GENUS V.-CORYNEUM, Kze. 1208. Coryneum pulvinatum, Kze. 1209. Coryneum disciforme, Kze. 1210. Coryneum compactum, B. \&

1211. Coryneum Kunzei, $C d$.

1212. Coryneum microstichum, $B$. $\& B r$

1213. Coryneum macrospermum, $B . \& B r$.

\section{GENUS VI. - PESTALOZZIA, D.Not.}

1214. Pestalozzia Guepini, Desm.

GENUS VII. - CHEIROSPORA, Er.

1215. Cheirospora botryospora, Fr.
GENUS VIII. NEMASPORA, $P$. 1216. Nemaspora crocea, $P$. 1217. Nemaspora rosæ, Desm.

GENUS IX.-MYXOSPORIUM, D. $N$.

1218. Myxosporium paradoxum, D. $N$.

1219. Myxosporium orbiculare, Berk.

1220. Myxosporium colliculosum, Berk.

\section{GENUS X.-GLGOSPORIUM,} Mlont.

1221. Glœosporium concentricum, B. \& Br.

1222. Glœosporium labes, $B . \& B r$. 1223. Glœosporium læticolor, Berk. 1224. Glœosporium fructigenum, Berk.

\section{ORdER XIV.-TORULACEI.}

GENUS I.-TORULA, $P$. 1225. Torula monilioides, $C d$. 1226. Torula ovalispora, Berk. 1227. Torula pulvillus, $B . \& B r$. 1228. Torula abbreviata, $C d$. 1229. Torula basicola, $B . \& B r$. 1230. Torula hysterioides, $C d$. 1231. Torula cylindrica, Berk. 1232. Torula eriophori, Berk.
1233. Torula herbarum, $L k$. 1234. Torula graminis, Desm. 1235. Torula plantaginis, $C d$. 1236. Torula sporendonema, $B . \&$ $B r$. GENUS II.-BACTRIDIUM, Kze. 1237. Bactridium flavum, Kze. 1238. Bactridium helvellæ, $B . \& B r$. 1239. Bactridium atrovirens, $B$. 
GENUS III. - HELICOSPORIUM, Nees.

1240. Helicosporium vegetum, Nees.

1241. Helicosporium pulvinatum, Fr.

GENUS IV.-BISPORA, $C d$.

1242. Bispora monilioides, $C d$.

GENUS $V$.-SEPTONEMA, $C d$.

1243. Septonema spilomeum, Berk.

1244. Septonema irregulare, Berk.

GENUS VI.-SPOROCHISMA, B. \& Br.

1245. Sporochisma mirabile, $B . \& B r$.

GENUS VII.-SPORIDESMIUM, $L k$.

1246. Sporidesmium polymorphum, $C d$.

1247. Sporidesmium antiquum, $C d$. 1248. Sporidesmium pyriforme, $C d$. 1249. Sporidesmium melanopum, $B . \& B r$.

1250. Sporidesmium scutellare, B. $\& B r$.
1251. Sporidesmium Lepraria, B. \& Br.

1252. Sporidesmium fungorum, Berk.

1253. Sporidesmium uniseptatum, B. \&Br.

GENUS VIII.-CONIOTHECIUM, $C d$.

1254. Coniothecium amentacearum, $C d$.

1255. Coniothecium betulinum, $C d$. GENUSIX.-DICTYOSPORIUM, $C d$.

1256. Dictyosporium elegans, $C d$.

GENUS $X$.-TETRAPLOA, $B . \& B r$.

1257. Tetraploa aristata, $B . \& B r$.

GENUS XI.-ECHINOBOTRYUM, $C d$.

1258. Echinobotryum atrum, $C d$.

\section{GENUS XII.-GYMNOSPO- RIUM, $C d$.}

1259. Gymnosporium arundinis, Cd.

\section{Order XV.-PUCCINIÆI.}

\section{GENUS I.-XENODOCHUS,} Schl.

1260. Xenodochus carbonarius, Schl.

GENUS II.-AREGMA, Fr. 1261. Aregma bulbosum, $F r$. 1262. Aregma gracile, Berk.
1263. Aregma mucronatum, Fr. 1264. Aregma acuminatum, $F r$. 1265. Aregma obtusatum, Fr.

GENUSIII.-TRIPHRA GMIUM, $L k$.

1266. Triphragmium ulmariæ, $L k$. 
GENUS IV.-PUCCINIA, $P$. 1267. Puccinia graminis, $P$. 1268. Puccinia striola, $L k$. 1269. Puccinia coronata, $\mathrm{Cd}$. 1270.' Puccinia truncata, $B . \& B r$. 1271. Puccinia asparagi, $D C$. 1272. Puccinia polygonorum, $I \%$. 1273. Puccinia vaginalium, $L /$. 1274. Puccinia primulæ, Grev. 1275. Puccinia veronicarum, $D C$. 1276. Puccinia glechomatis, $D C$. 1277. Puccinia menthæ, $P$. 1278. Puccinia scorodoniæ, $L k$. 1279. Puccinia scrophulariæ, Lib. 1280. Puccinia betonicæ, $D C$. 1281. Puccinia vincæ, Berk. 1282. Puccinia campanulæ, Carm. 1283. Puccinia clandestina, Carm. 1284. Puccinia compositarum, Schl. 1285. Puccinia syngenesiarum, $L k$. 1286. Puccinia glomerata, Grev. 1287. Puccinia variabilis, Grev. 1288. Puccinia valantiæ, $P$. 1289. Puccinia galiorum, $L k$. 1290. Puccinia umbelliferarum,

1251. Puccinia ægopodii, $I k$. 1292. Puccinia saniculæ, Grev. 1293. Puccinia bullaria, $L k$. 1294. Puccinia smyrnii, $C d$. 1295. Puccinia anemones, $P$. 1296. Puccinia calthæ, $L k$. 1297. Puccinia violarum, $L k$. 1298. Puccinia lychnidearum, $L k$. 1299. Puccinia umbilici, Guép.
1300. Puccinia rhodiolæ, B. \& $\mathrm{Br}$. 1301. Puccinia saxifragarum, Schl. 1302. Puccinia chrysosplenii, Grev. 1303. Puccinia epilobii, $D C$. 1301. Puccinia pulverulenta, Grev. 1305. Puccinia circææ, $P$. 1306. Puccinia prunorum, $L /$. 1307. Puccinia fabæ, $L /$. 1308. Puccinia buxi, $D C$.

\section{GENUS V.-GYMNOSPORAN- GIUIM, $D C$.}

1309. Gymnosporangium juniperi, $L k$.

GENUS VI.-PODISOMA, Lk.

1310. Podisoma juniperi, $F r$.

1311. Podisoma foliicolum, Bert.

1312. Podisoma sabinæ, Fr.

GENUS VII.-UREDO, Lév.

1313. Uredo potentillarym, $D C$.

1314. Uredo saxifragarum, $D C$.

1315. Uredo filicum, Desm.

1316. Uredo pustulata, $P$.

1317. Uredo hypericorum, $D C$.

1318. Uredo caryophyllacearum, Johnst.

1319. Uredo quercus, Brond.

1320. Uredo vacciniorum, $P$. 1321. Uredo confluens, $P$. 1322. Uredo alliorum, $D C$. 1323. Uredo statices, Desm. 1324. Uredo circææ, A. \& S. 1325. Uredo bifrons, Grev. 
GENUS VIII.-TRICHOBASIS, Lév.

1326. Trichobasis rubigo-vera, Lév.

1327. Trichobasis linearis, Lév.

1328. Trichobasis glumarum, Lév. 1329. Trichobasis symphyti, Lév. 1330. Trichobasis pyrolæ, Berk. 1331. Trichobasis petroselini, Berk. 1332. Trichobasis senecionis, Berk. 1333. Trichobasis caricina, Berk. 1334. Trichobasis oblongata, Berk. 1335. Trichobasis scillarum, Berk. 1336. Trichobasis cichoracearum,

1337. Trichobasis artemisiæ, Berk. 1338. Trichobasis labiatarum, Lév. 1339. Trichobasis 1ychnidearum, Lév.

1340. Trichobasis umbellatarum, Lév.

1341. Trichobasis heraclei, Berk.

1342. Trichobasis betæ, Lév.

1343. Trichobasis fabæ, Lév.

1344. Trichobasis galii, Lév.

1345. Trichobasis suaveolens, Lév.

1346. Trichobasis polygonorum,

1347. Trichobasis vincæ, Berk.

1348. Trichobasis geranii, Bert.

1349. Trichobasis violarum, Berk.

1350. Trichobasis epilobii, Bèrk.

GENUS IX.-UROMYCES, Lév.

1351. Uromyces alliorum, $D C$.

1352. Uromyces ulmariæ, Lév.

1353. Uromyces appendiculata,
1354. Uromyces apiculata, Lév. 1355. Uromyces limonii, Lév. 1356. Uromyces ficariæ, Lév. 1357. Uromyces primulæ, Lév. 1358. Uromyces intrusa, Lév. 1359. Uromyces iridis, Lév.

\section{GENUS X.-COLEOSPORIUM, Lév.}

1360. Coleosporium tussilaginis, Lév.

1361. Coleosporium pingue, Lév.

1362. Coleosporium petasites, Lév.

1363. Coleosporium campanulæ, Lév.

1364. Coleosporium sonchi-arvensis, Lév.

1365. Coleosporium rhinanthacearum, Lév.

\section{GENUS XI.-MELAMPSORA, Cast.}

1366. Melampsora euphorbiæ, Cast. 1367. Molampsora populina, Lév. 1368. Melampsora tremulæ, Tul. 1369. Melampsora betulina, Desm. 1370. Melampsora salicina, Lév.

GENUS XII.-LECYTHEA, Lév. 1371. Lecythea ruborum, Lév.

1372. Lecythea rosæ, Lév. 1373. Lecythea poterii, Lév. 1374. Lecythea populina, Lév. 1375. Lecythea euphorbiæ, Lév. 1376. Lecythea epitea, Lév. 1377. Lecythea mixta, Lév. 1378. Lecythea saliceti, Lév. 1379. Lecythea Baryi, Berk. 1380. Lecythea valerianæ, Berk. 
1381. Lecythea gyrosa, Lév.

1382. Lecythea capræarum, Lév.

1383. Lecythea lini, Lév.

GENUS XIII.-CYSTOPUS, Lév.

1384. Cystopus candidus, Lév.

\section{GENUS XIV.-POLYCYSTIS, Lév.}

1385. Polycystis colchici, Tul.

1386. Polycystis violæ, B.\& Br.

1387. Polycystis pompholygodes, Lév.

1388. Polycystis parallela, $B . \& B r$.

GENUS XV.-TILLETIA, Tul. 1389. Tilletia caries, $T u l$.

GENUS XVI.-USTILAGO, $L k$. 1390. Ustilago segetum, Ditm.

1391. Ustilago urceolorum, Tul.
1392. Ustilago longissima, Tul. 1393. Ustilago olivacea, Tul. 1394. Ustilago hypodytes, Fr. 1395. Ustilago maydis, $C d$. 1396. Ustilago Montagnei, Tul. 1397. Ustilago typhoides, B. \& Ir. 1398. Ustilago Salveii, $B . \& B r$. 1399. Ustilago grammica, B. \& $\mathrm{Br}$. 1400. Ustilago vinosa, Tul. 1401. Ustilago utriculosa, Tul. 1402. Ustilago flosculorum, Tul. 1403. Ustilago receptaculorum, Fr. 1404. Ustilago antherarum, Tul. GENUS XVII.-TUBURCINIA, Fr.

1405. Tuburcinia scabies, Berk. 1406. Tuburcinia trientalis, B. \& $B r$.

\section{ORder XVI.-\#ECIDIACEI.}

GENUS I.-REETELIA, Reb. 1407. Rœstelia cancellata, Reb. 1408. Rœstelia cornuta, Tul. 1409. Rœstelia lacerata, Tul.

\section{GENUS II.-PERIDERMIUM, Chev.}

1410. Peridermium pini, Chev.

1411. Peridermium elatinum, $L k$.

GENUS III.-ÆCIDIUM, Pers.

a Sparsa.

1412. Æcidium leucospermum,
1413. Æcidium quadrifidum, $D C$. 1414. Æcidium albescens, Grev. 1415. \#scidium epilobii, $D C$. 1416. झtcidium soldanellæ, Hornsch.

1417. 环cidium tragopogonis, Pers. 1418. 坬cidium euphorbiæ, Pers.

\section{$\beta$ Elongata.}

1419. 拨cidium berberidis, Pers. 1420. झৈcidium thalictri, Grev. 1421. 环cidium crassum, Pers. 1422. 珑idium periclymeni, $D C$. 
$\gamma$ Poculiforme.

1423. Fcidium calthæ, Grev.

1424. झcidium ranunculacearum,

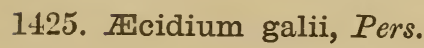

1426. 妮cidium bunii, $D C$.

1427. Fidium valerianacearum,

Dub.

1428. \#cidium asperifolii, Pers.

1429. 死cidium grossulariæ, $D C$.

1430. झcidium urticæ, $D C$.

1431. Eॄcidium Behenis, DC.

1432. FEcidium orobi, $D C$.

$\delta$ Sub-immersce.

1433. झcidium compositarum, ILart.

1433^. Frcidium compositarum, var. taraxaci, Grev.

1433в. FIsidium compositarum, var. prenanthis, Pers.

1433c. \#cidium compositarum, var. tussilaginis, Pers.
1433D. Fiscidium compositarum, var. Jacobeæ, Grev.

1433E. \#scidium compositarum, var. lapsani, Purt.

1434. झEcidium saniculæ, Carm.

1435. झtcidium violæ, Schum.

1436. \#تcidium poterii, Cooke.

1437. झलidium geranii, $D C$.

1438. झ世cidium menthæ, $D C$.

1439. झcidium pedicularis,

Lobosch.

1440. 佂cidium primulæ, $D C$.

1441. Wridium rubellum, Pers.

1442. Fiscidium ari, Berk.

1443. \#cidium allii, Berk.

1444. FEcidium dracontii, Schw.

GENUS IV.-ENDOPHYLLUM, Lév.

1445. Endophyllum sempervivi, Lév. 


\section{FAMILY IV.-HYPHOMYCETES.}

\section{ORDER XVII.-ISARIACEI.}

GENUS I.-ISARIA, IIill.

1446. Isaria farinosa, $F r$.

1447. Isaria arachnophila, Ditm.

1448. Isaria brachiata, Schum.

1449. Isaria citrina, $P$.

1450. Isaria intricata, $\mathrm{Fr}$.

1451. Isaria Friesii, MIont.

1452. Isaria puberula, Berk.

GENUS II.-ANTHINA, $F r$. 1453. Anthina flammea, Fr.
GENUS III.-CERATIUM, A. \& $S$.

1454. Ceratium hydnoides, $A . \& S$. GENUS IV.-PACHNOCYBE, Berk.

1455. Pachnocybe subulata, Eerk. 1456. Pachnocybe grisea, Berk. 1457. Pachnocybe acicula, Berk. 1458. Pachnocybe albida, Berk.

DOUBTFUL SPECIES.

Anthina flavovirens, $F r$.

Anthina brunnea, $F r$.

\section{ORder XVIII.-STILBACEI.}

GENUS I.-STILBUM, Tode. 1459. Stilbum tomentosum, Schrad. 1460. Stilbum aurantiacum, Berk. 1461. Stilbum vaporarium, B.\& Br. 1462. Stilbum fasciculatum, B. \& Br.

1463. Stilbum fimetarium, $B . \& B r$. 1464. Stilbum erythrocephalum, Ditm.

1465. Stilbum rigidum, $P$.

1466. Stilbum piliforme, $P$.

1467. Stilbum bicolor, $P$.

1468. Stilbum anomalum, Berk.
1469. Stilbum nigrum, Berk.

1470. Stilbum pellucidum, Schrad.

1471. Stilbum turbinatum, Tode.

1472. Stilbum vulgare, Tode.

GENUS II.-VOLUTELIA, Tode. 1473. Volutella ciliata, $F r$.

1474. Volutella setosa, Berk.

1475. Volutella hyacinthorum, Berk.

GENUS III.-TUBERCULARIA, Tode.

1476. Tubercularia vulgaris, Tode.

1477. Tubercularia granulata, $P$. 
1478. Tubercularia nigricans, $L k$. 1479. Tubercularia persicina,Ditm.

GENUS IV.-FUSARIUM, $L k$. 1480. Fusarium lateritium, Nees. 1481. Fusarium tremelloides, Grev. 1482. Fusarium rossum, $L 7$.

14S3. Fusarium heterosporium, $N$.

$$
\begin{gathered}
\text { GENUS V.-MYROTHECIUM, } \\
\text { Tode. }
\end{gathered}
$$

1484. Myrothecium roridum, Tode.

GENUS VI.-EPICOCCUM, $L k$. 1485. Epicoccum neglectum, Desm. 1486. Epicoccum equiseti, Berk.
GENUS VII.-ILLOSPORIUM, Mart.

1487. Illosporium roseum, Fr. 1488. Illosporium carneum, Frr. 1489. Illosporium corallinum, Roberge.

1490. Illosporium coccineum, $F r$.

GENUS VIII.-IEGERITA, $P$. 1491. 开gerita candida, $P$.

\section{DOUBTFUL SPECIES.}

Atractium flammeum, B. \& Rav. Volutella Buxi, B. \& Br .

Volutella melaloma, $B . \& B r$.

\section{- Order XIX.-DEMatiei.}

GENUS I.-ARTHROBOTRYUM Ces.

1492. Arthrobotryum atrum, B. \& Br.

1493. Arthrobotryum stilboideum, Ces.

\section{GENUS II.-DENDRYPHIUM, $C d$.}

1494. Dendryphium comosum, Walr

1495. Dendryphium curtum,

1496. Dendryphium laxum,

B. \& Br.

1497. Dendryphium griseum,

1498. Dendryphium fumosum, B. $\& B r$. Beric.

GENUS III.-PERICONIA, $C l$. 1499. Periconia glaucocephala, $\mathrm{Cl}$.
1500. Periconia calicioides, Berk. GENUS IV.-SPOROCYBE, Fr. 1501. Sporocybe byssoides, Fr. 1502. Sporocybe nigrella, Berk. 1503. Sporocybe alternata, Berk. GENUS $\quad$.-STACHYBOTRYS, $C d$.

1504. Stachybotrys atra, $C d$. 1505. Stachybotrys lobulata, Derl. GENUS VI.-HAPLOGRAPHIUM B. \& Br.

1506. Haplographium delicatum, B. \& Br.

GENUS VII.-MONOTOSPORA, $C d$.

1507. Monotospora megalospora, B. \& Br. 1508. Monotospora sphærocephala, B. \& Br. 
GENUS VIII.-CEPHALOTRICHUM, $L k$.

1509. Cophalotrichum curtum, Berk.

GENUS IX.-CEDEMIUM, Fr. 1510. CEdemium atrum, $F r$.

\section{GENUS $X$.-HELICOCORYNE, $C d$.}

1511. Helicocoryne viridis, $C d$.

$G E N U S X I$ - HELMINTHOSPORIUII, $L k$.

1512. Helminthosporium Smithii, B. \& Br.

1513. Helminthosporium folliculatum, $C d$.

1514. Helminthosporium macrocarpum, Grev.

1515. Helminthosporium subulatum, Nees.

1516. Helminthosporium velutinum, $L \%$.

1517. Helminthosporium fusisporium, Berk.

1518. Helminthosporium nanum, Nees.

1519. Helminthosporium simplex,

1520. Helminthosporium tiliæ, Fr.

1521. Helminthosporium Roussellianum, Mont.

1522. Helminthosporium turbinatum, $B . \& B r$.

1523. Helminthosporium obovatum, Berk.

1524. Helminthosporium delicatulum, Berk.

1525. Helminthosporium sticticum $B . \& B r$.

1526. Helminthosporium clavariarum, Desm.

1527. Helminthosporium oosporum, $C d$.
1528. Helminthosporium apicula. tum, $C d$.

1529. Helminthosporium dendroideum, Berk.

1530. Helminthosporium apicale, Berk.

1531. Helminthosporium altum, Preuss.

GENUS XII.-MACROSPORIUII Fr.

1532. IIacrosporium cheiranthi, Fr.

1533. IIacrosporium sarcinula, Berte.

1534. Macrosporium concinnum, Berk.

1535. Macrosporium brassicæ, Bert.

GENUS XIII.-MYSTROSPO. RIUM, $C d$.

1536. IIystrosporium stemphylium, $C u^{\prime}$.

GENUS XIV.-ACROTHECIUI, $C d$.

1537. Acrothecium simplex, $B$.

GENUS XV.-TRIPOSPORIUM, $C d$.

1538. Triposporium elegans, $C d$.

GENUS XVI.-HELICOMA, $C d$. 1539. Helicoma Mulleri, $C d$.

GENUS XVII.-CLADOTRICHUM, $C d$.

1540. Cladotrichum triseptatum, B. \& Br.

GENUS XVIII.-POLYTHRINCIUM, Kze.

1541. Polythrincium trifolii, Kze.

GENUS XIX.-CLADOSPORIUM, $L k$.

1542. Cladosporium herbarum, $L k$. 
INDEX FUNGORUM BRITANNICORUM.

1543. Cladosporium dendriticum, Walr.

1543^. Cladosporium dendriticum, var. $\beta$ orbiculatum, Desm.

1544. Cladosporium depressum, B. $\& B r$.

1545. Cladosporium brachormium, B. \&Br.

1546. Cladosporium lignicolum, $C d$.

1547. Cladosporium nodulosum, $C l$.

GENUS XX.-ARTHRINIUM,

1548. Arthrinium sporophleum,
GENUS XXI.-GONATOSPORIUM, $C d$.

1519. Gonatosporium puccinioides Cd. GENUS XXII.- CAMPTOUM,

1550. Camptoum curvatum, $L k$.

GENUS XXIII.-SPORODUM,

1551. Sporodum conopleoides, $C d$.

DOUBTFUL SPECIES.

Cladosporium fumago, $L /$.

Actinonema cratægi, Pers.

\section{Order XX.-MUCEDINES.}

GENUS I.-ASPERGILIUS, Mich.

1552. Aspergillus glaucus, $L k$.

1553. Aspergillus dubius, $C d$.

1554. Aspergillus candidus, $L k$.

1555. Aspergillus roseus, $L k$.

1556. Aspergillus mollis, Berk.

1557. Aspergillus virens, $L k$.

\section{GENUS II.-NEMATOGONUM,} Desm.

1558. Nematogonum aurantiacum,

1559. Nematogonum aureum, Berk.

GENUS III.-RHINOTRICHUM $C d$.

1560. Rhinotrichum Bloxami, B. \& Br.
1561. Rhinotrichum Thwaitesii, B. $\& B r$.

1562. Rhinotrichum Opuntia,

B. \& Br.

GENUS IV.-BOTRYTIS, Nich. 1563. Botrytis Tilletii, Desm. 1564. Botrytis citrina, Berk. 1565. Botrytis Jonesii, $B . \& B r$. 1566. Botrytis terrestris, $P$.

GENUS $V$.-ACROSPEIRA, B. \& $B r$.

1567. Acrospeira mirabilis, $B . \& \mathrm{Br}$. GENUS VI.-PERONOSPORA,
Casp.

1568. Peronospora parasitica, $C d$. 1569. Peronospora destructor, Casp. 
1570. Poronospora infestans, Casp. 1571. Poronospora ganglioniformis Casp.

1572. Poronospora arborescens, Casp.

1573. Peronospora viciæ, Casp. 1574. Peronospora urticæ, Casp. 1575. Poronospora arenariæ, Casp. 1576. Peronospora macrospora, Casp.

1577. Peronospora grisea, Unger.

1578. Peronospora effusa, Casp.

1579. Peronospora violacea, Berk. 1580. Peronospora curta, Casp.

1581. Peronospora sordida, B. \& Br. 1582. Peronospora sparsa, Berk.

GENUS VII.-VERTICILLIUN, $L k$.

1583. Verticillium apicale,

1584. Verticillium nanum, B. $\& B r$. B. \& Br.

1585. Verticillium epimyces, B. $\& B r$.

1586. Verticillium distans, B.\& Br.

GENUS VIII.-HAPLARIA, $L k$. 1587. Haplaria grisea, $L k$.

GENUS IX.-POLYACTIS, $L k$. 1588. Polyactis vulgaris, $L \%$.

1589. Polyactis cana, Berk.

1590. Polyactis vera, Berk.

1591. Polyactis cinerea, Berk.

1592. Polyactis fasciculata, $\mathrm{Cd}$.
GENUS $X_{0}$-PENICILLIUII, $L k$ :

1593. Penicillium crustaceum, Fr. 1594. Penicillium sparsum, Grev. 1594. Ponicillium sparsum, var. $\beta$ coremium, Grev.

1595. Penicillium bicolor, Fr. 1596. Penicillium candidum, $L k$. 1597. Penicillium roseum, $L k$. 1598. Penicillium subtile, Berk. GENUS XI.-OIDIUM, Llc. 1599. Oidium chartarum, $L k$. 1600. Oidium aureum, $L k$. 1601. Oidium fulvum, $L k$. 1602. Oidium fructigenum, Schrad. 1603. Oidium fasciculatum, Berk. 1604. Oidium porriginis, MLont. 1605. Oidium favorum, $B . \& B r$. 1606. Oidium æquivocum, $B . \& B r$. 1607. Oidium concentricum, B. $\& B r$. 1608. Oidium abortifaciens, $B$. GENUS XII.-MONILIA, Hill. 1609. Monilia fasciculata, $C d$. 1610. Monilia racemosa, Purt. GENUS XIII.-CYLINDRIUM,
Bon. 1611. Cylindrium septatum, Bon. $G E N U S$ XIV.-DACTYLIUN,
Nees. 1612. Dactylium pyriferum, $F r$. 1613. Dactylium tenollum, Fr. 
1614. Dactylium macrosporum, Fr. 1615. Dactylium sphærocephalum, Berk.

1616. Dactylium dendroides, Fr.

1617. Dactylium obovatum, Berk.

1618. Dactylium roseum, Berk.

GENUS $X V$.-FUSIDIUM, $L k$.

1619. Fusidium griseum, $L k$.

1620. Fusidium flavovirens, $F r$.

1621. Fusidium album, Desm.

\section{GENUS XVI.-SPOROTRI- CHUM, $L k$.}

1622. Sporotrichum chlorinum, Ik.

1623. Sporotrichum aurantiacum, Grev.

1624. Sporotrichum sulphureum, Grev.

1625. Sporotrichum laxum, Grev.

1626. Sporotrichum inosculans, Berk.

1627. Sporotrichum fenestrale, Ditm.

GENUS XVII.-ZYGODESMUS,

1628: Zygodesmus fuscus, $C d$.

GENUS XVIII.-VIRGARIA, Nees.

1629. Virgaria nigra, Fr.

GENUS XIX.-BOLACOTRICHA B. \& Br.

1630. Bolacotricha grisea, $B . \& B r$.

GENUS XX.-MYXOTRICHUM, Kze.

1631. Myxotrichum chartarum, Kze.

1632. Myxotrichum deflexum, Berk.
GENUS XXI.-GONYTRICHUM Nees.

1633. Gonytrichum cæsium, Nees.

GENUS XXII.-MENISPORA, $P$. 1634. Menispora lucida, $C d$.

GENUS XXIII-CHATOPSIS, Grev.

1635. Chætopsis Wauchii, Grev.

GENUS XXIV.-ACREMONIUM $L k$.

1636. Acremonium verticillatum, $L k$.

1637. Acremonium alternatum, $L k$. 1638. Acremonium fuscum, $\$ c h m$.

\section{GENUS $X X V$.-GONATOBO-} TRYS, $C d$.

1639. Gonatobotrys simplez, $C d$.

GENUS XXVI-CLONOSTACHYS, $C d$.

1640. Clonostachys araucaria, $C d$.

GENUS XXVII.-BOTRYOSPORIUII, $C d$.

1641. Botryosporium pulchrum, $C d$.

1642. Botryosporium diffusum, $C d$.

GENUS XXVIII.-PAPULASPORA, Preuss.

1643. Papulaspora sepedonioides, Preuss.

GENUS XXIX.-RHOPALOMYCES, $C d$.

1644. Rhopalomyces pallidus, B. \& Br.

1645. Rhopalomyces candidus, B. \&Br. 
DOUBTFUI, OR FX'IINC'T SPECIES.

Verticillium lateritium, Rabh. Penicillium fasciculatum, Somm.

' Oidium Tuckeri, Berk.

Oidium balsamii.
Oidium erysiphoides, $F r$.

Oidium leucoconium, Desm.

Oidium monilioides, $L \%$.

Dactylium tenuissimum, Berk.

Sporotrichum geochroum, Desm.

\section{Order XXI.-SEPEDONIEI.}

GENUS I.-SEPEDONIUM, $L k$. 1646. Sepedonium chrysospermum 1647. Sepedonium roseum, $F r$. GENUS II.-FUSISPORIUM, $L k$. 1648. Fusisporium betæ, Desm. 1649. Fusisporium Georginæ,Berk. 1650. Fusisporium udum, Berk. 1651. Fusisporium roseolum, Steph. 1652. Fusisporium fœni, $B . \& B r$. 1653. Fusisporium bacilligerum,

1654. Fusisporium atrovirens,
1655. Fusisporium aurantiacum Ll.

1656. Fusisporium incarcerans, Berk.

1657. Fusisporium (?) insidiosum, Berk.

1658. Fusisporium (?) solani tuberosi, MLart.

GENUS III.-EPOCHNIUM, $L k$. 1659. Epochnium macrosporoideum, Ber\%.

GENUS IV.-PSILONIA, Fr. 1660. Psilonia nivea, Fr. 1661. Psilonia gilva, $\mathrm{Fr}$. 1662. Psilonia arundinis, Desm.

\section{ORDER XXII.-TRICHODERMACEI.}

GENUS I.-PILACRE, Fr. 1663. Pilacre faginea, $B . \& B r$. 1664. Pilacre Petersii, B. \& Curt.

GENUS II.-INSTITALE, Fr. 1665. Institale effusa, $F r$.

GENUS III.-TRICHODERMA, $P$.

1666. Trichoderma viride, $P$.
GENUS IV.-ARTHRODERMA, Curr.

1667. Arthroderma Curreyi, Berk.

DOUBTFUL OR EXTINCT SPECIES.

Institale acariformis, $F r$.

Institale radiata, $\mathrm{Fr}$. 


\section{FAMILY V.-ASCOMYCETES.}

\section{Order XXIII.-ELVELLACEI.}

GENUS I.-MORCHELLA, Dill. 1668. Morchella esculenta, Pers. 1669. Morchella patula, Pers. 1670. Morchella semilibera, $D C$.

GEITUS II.-GYROMITRA, Fr. 1671. Gyromitra esculenta, $F r$. GENES III.-HELVELLA, Linn. 1672. Holvella gigas, Kromb. 1673. Helvella crispa, $F r$. 1674. Helvella lacunosa, Afz. 1675. Helvella elastica, Bull. 1676. Helvella ephippium, Lev.

GENUS IV.-VERPA, Swartz. 1677. Verpa digitaliformis, Pers. 1678. Verpa conica, Sow.

GENUS V.-MITRULA, Fr. 1679. Mitrula cucullata, Fr. 1680. Mitrula paludosa, Fr.

\section{GENUS VI.-SPATHULARIA, Pers.}

1681. Spathularia flavida, Pers.

GENUS VII.-LEOTIA, Hill. 1682. Leotia lubrica, Pers.

GENUS VIII.-VIBRISSEA, Fr. 1683. Vibrissea truncorum, $F r$.

\section{GENUS IX.-GEOGLOSSUM, Pers.}

1684. Geoglossum viride, $P$.

1685. Geoglossum olivaceum, $P$. 1686. Geoglossum glutinosum, $P$. 1687. Geoglossum viscosum, $P$. 1688. Geoglossum glabrum, $P$. 1689. Geoglossum hirsutum, $P$. 1690. Geoglossum difforme, $F r$.

GENUS X.-PEZIZA, Linn.

$$
\text { A.-Aleuria, } F r \text {. }
$$

Sub-Genus 1.-DISCINA, $\mathrm{Fr}$. 1691. Peziza acetabulum, $L$.

1692. Peziza venosa, $P$.

1693. Peziza badia, $P$.

1694. Peziza leporina, Batsch.

1695. Peziza onotica, $P$.

1696. Peziza aurantia, Fr.

1697. Peziza fibrillosa, Curr.

1698. Peziza cochleata, Huds.

1699. Peziza succosa, $B$.

1700. Peziza repanda, Wahl.

1701. Peziza cerea, Sow.

1702. Peziza vesiculosa, Bull.

1703. Peziza micropus, $P$.

1704. Peziza pustulata, $P$.

1705. Peziza radula, $B$. \& $B r$. 
1706. Peziza viridaria, B.\& Br.

1707. Peziza luteo nitens, B. \& Br.

Sub-Genus 2.-GEOPYXIS, Fr.

1708. Peziza macropus, $P$.

1709. Peziza tuberosa, Bull.

1710. Peziza cupularis, $L$.

1711. Peziza sepulta, Fr.

1712. Peziza cornubiensis, B.\& Br.

1713. Peziza saniosa, Schrad.

1714. Peziza granulata, Bull.

1715. Peziza geaster, $B . \& B r$.

Sub-Genus 3.-HUMARIA, Fr. 1716. Peziza rutilans, $\mathrm{Fr}$.

1717. Peziza melaloma, $A . \& S$.

1718. Peziza erecta, Sow.

1719. Peziza polytrichi, Sch.

1720. Peziza leucoloma, Reb.

1721. Peziza humosa, Fir.

1722. Peziza glumarum, Desm.

1723. Peziza omphaloides, Bull.

Sub-Genus 4.-ENCOELIA, Fr. 1724. Peziza fascicularis, $A$. \& $S$. 1725. Peziza furfuracea, $F r$.

$$
\text { 23.-LACHNEA, Fr. }
$$

Sub-Genus 5.-SARCOSCYPHA, Fr. 1726. Peziza coccinea, Jacq.

1727. Peziza melastoma, Sow. 1728. Peziza radiculata, Sow.

1729. Peziza hemispherica, Wigg.

1730. Peziza brunnea, A.\& S.

1731. Peziza hirta, Sch.

1732. Peziza trechispora, $B . \& B r$.

1733. Peziza vitellina, $P$.

1734. Peziza scutellata, $L$.
1735. Peziza cærulea, Bolt.

1736. Peziza livida, Sch.

1737. Peziza stercorea, $P$.

1738. Peziza theleboloides, A. \& S. 1739. Peziza albo-spadicea, Grev.

Sub-Genus 6.-DASYSCYPHA, Fr. 1740. Peziza ciliaris, Schr.

1741. Peziza virginea, Batsch.

1742. Peziza nivea, $\mathrm{Fr}$.

1743. Peziza calycina, Schum.

1744. Peziza bicolor, Bull.

1745. Peziza cerinea, $P$.

1746. Peziza clandestina, Bull.

1747. Peziza caulicola, $\mathrm{Fr}$.

1748. Peziza acuum, Fr.

1749. Peziza albo-violascens, A. $\& S$.

1750. Peziza corticalis, $P$.

1751. Peziza tricolor, Sow.

1752. Peziza melaxantha, Fr.

1753. Peziza hispidula, Schr.

1754. Peziza Schumacheri, Fr.

1755. Peziza rufo-olivacea, $A$. \& $S$.

1756. Peziza diplocarpa, Curr.

1757. Peziza variecolor, $F r$.

1758. Peziza episphæria, Mart.

1759. Peziza spineti, Batsch.

1760. Peziza papillaris, Bull.

1761. Peziza hyalina, $P$.

1762. Peziza sulphurea, $P$.

1763. Peziza plano-umbilicata,

Grev.

1764. Peziza villosa, $P$.

1765. Peziza Grevillei, $B$.

1766. Peziza Berkeleii, Blox. 
1767. Peziza aspidiicola, $B . \& B r$.

1768. Peziza albo-testacea, Desm.

1769. Peziza apala, B.\&Br.

1770. Peziza clavariarum, Desm.

1771. Peziza straminum, $B . \& B r$. 1772. Peziza vectis, $B . \& B r$.

1773. Peziza ilicincola, $B . \& B r$. ' 1774. Peziza calyculæformis, Schum.

Sub-Genus 7.-TAPESIA, $F r$.

1775. Peziza anomala, $P$.

1776. Peziza aurelia, $P$.

1777. Peziza domestica, Sow.

1778. Peziza Piggotii, B. \& Br.

1779. Peziza cæsia, $P$.

1780. Peziza Chavetiæ, Lib.

1781. Peziza eriobasis, $B$.

1782. Peziza Bloxami, B. \& Br.

1783. Peziza mutabilis, $B . \& B r$.

1784. Peziza rosæ, $P$.

1785. Peziza fusca, $P$.

1786. Peziza Johnstoni, $B$.

1787. Peziza sanguinea, $P$.

Sub-Genus 8.-FIBRINA, Fr. 1788. Peziza rudis, $B$.

1789. Peziza bolaris, Batsch.

1790. Peziza siparia, $B . \& B r$.

1791. Peziza ledi, $A . \& S$.

$$
\text { C.-Phialea, Fr. }
$$

Sub-Genus 9.-HYMENOSCYPHA, Fr.

1792. Peziza firma, $P$.

1793. Peziza ciborioides, $F r$.

1794. Peziza echinophila, Bull.
1795. Peziza coronata, Bull.

1796. Peziza inflexa, Bolt.

1797. Peziza Persoonii, Mloug.

1798. Peziza striata, Fr.

1799. Peziza cacaliæ, $\mathrm{Fr}$.

1800. Peziza nitidula, $B . \& B r$.

1801. Peziza cyathoidea, Bull.

1802. Peziza caucus, Reb.

1803. Peziza Curreyi, $B$.

Sub-Genus 10.-MOLLISIA, Fr.

1804. Peziza clavus, $A$. \& $S$.

1805. Peziza vinosa, $A$. \& $S$.

1806. Peziza atrovirens, $P$.

1807. Peziza cinerea, Batsch.

1808. Peziza Chailletii, $P$.

1809. Peziza sphærioides, $P$.

1810. Peziza axillaris, Nees.

1811. Peziza xanthostigma, Fr.

1812. Peziza leucostigma, Fr.

1813. Peziza vulgaris, Fr.

1814. Peziza erumpens, Grev.

1815. Peziza atrata, $P$.

1816. Peziza cornea, $B . \& B r$.

1817. Peziza fusarioides, $B$.

1818. Peziza micrometra, B. \&Br.

1819. Peziza paulula, Rob.

1820. Peziza lacustris, Fr.

Sub-Genus 11.-PATELLEA, Fr.

1821. Peziza melanotheja, Fr.

1822. Peziza compressa, A. \& S.

1823. Peziza lignyota, $F r$.

1824. Peziza flexella, Fr.

1825. Peziza lecideola, Fr. 
GENUS XI.-HELOTIUM, $F r$.

Sub-Genus 1.-PELASTEA, Fr. 1826. Holotium fibuliformo, $\mathrm{Fr}$. 1827. Helotium agaricinum, $B$. 1828. Helotium sclerotioides, $B$. 1829. Helotium aciculare, $\mathrm{Fr}$. 1830. Helotium subtile, $\mathrm{Fr}$. 1831. Helotium æruginosum, $F r$. 1832. Helotium serotinum, $\mathrm{Fr}$. 1833. Helotium virgultorum, $F r$. 1833^. Helotium virgultorum. var. $\beta$ flavescens, Holms.

1834. Helotium lutescens, $\boldsymbol{F r}$. 1835. Helotium testaceum, $B$. 1836. Helotium conigenum, $F r$. 1837. Helotium phascoides, $\mathbb{F r}$. 1838. Helotium acuum, $\mathrm{Fr}$. 1839. Helotium luteolum, Curr. 1840. Helotium aquaticum, Curr.

Sub-Genus 2.-CALYCELLA, Fr. 1841. Helotium tuba, $F r$.

1842. Helotium buccina, $F r$. 1843. Helotium calyculus, $\mathrm{Fr}$. 1844. Helotium Aspegrenii, Fr. 1845. Helotium citrinum, $\mathbb{F r}$. 1846. Helotium pallescens, $F r$. 1847. Helotium lenticulare, $F r$. 1848. Helotium ochraceum, $B$. 1849. Helotium imberbe, $\mathrm{Fr}$. 1850. Helotium cribrosum, $B$. 1851. Helotium claro-flavum, $B$. 1852. Helotium salicellum, $\mathrm{Fr}$. 1853. Helotium versiforme, $F r$. 1854. Helotium subsessile, Sch.
1855. Helotium herbarum, $F r$. 1856. Helotium epiphyllum, Fr. 1857. Helotium fagineum, $F r$. 1858. Helotium punctatum, Fr. 1859. Helotium marchantiæ, $F r$. 1860. Helotium infundibulum, $F r$. 1861. Helotium ferrugineum, $\mathrm{Fr}$. GENUS XII.-PSILOPEZIA, $B$. 1862. Psilopezia Babingtonii, $B$.

\section{GENUS XIII.-PATELLARIA, Fr.}

1863. Patellaria atrata, $F r$. 1864. Patellaria rhabarbarina, $P$. 1865. Patellaria citrina, B. \& Br. 1866. Patellaria clavispora, B. $\& B r$. 1867. Patellaria livida, B. \& Br. 1868. Patellaria discolor, Mont. 1869. Patellaria proxima, B. \& Br. 1870. Patellaria atrovinosa, Blox. 1871. Patellaria aquatica, Curr. 1872. Patellaria palustris, Curr.

\section{GENUS XIV.-SPHINCTRINA, $\mathrm{Fr}$.}

1873. Sphinctrina turbinata, $F r$. GENUS $X V$.-LAQUEARIA, $F r$. 1874. Laquearia sphæralis, $F r$.

\section{GENUS XVI.-TYMPANIS, Tode.}

1875. Tympanis alnea, $P$. 1876. Tympanis fraxini, Schw. 1877. Tympanis conspersa, Fr. 1878. Tympanis saligna, Tode. 
GENUS XVII.-CENANGIUM, Fr.

1879. Cenangium ribis, $F r$.

1880. Cenangium cerasi, $F r$.

1881. Cenangium prunastri, Fr.

1882. Cenangium aucupariæ, Fr.

1883. Cenangium pulveraceum, Fr.

1884. Cenangium fuliginosum, $F r$.

1S85. Cenangium ferruginosum, Fr.

1886. Cenangium quercinum, $F r$.

1887. Cenangium rubi, Fr.

- GENUS XVIII.-ASCOBOLUS, Tode.

1888. Ascobolus furfuracens, $P$. 1889. Ascobolus viridis, Curr. 1890. Ascobolus vinosus, $B$. 1891. Ascobolus ciliatus, Schm. 1892. Ascobolus glaber, $P$. 1893. Ascobolus trifolii, Bernh. 1894. Ascobolus carneus, $P$. 1895. Ascobolus saccharinus, B. \& Curr.

GENUS XIX.-BULGARIA, Fr. 1896. Bulgaria inquinans, $F r$. 1897. Bulgaria sarcoides, $F r$.

$$
\text { GENUS XX.-AGYRIUM, } F r \text {. }
$$

1898. Agyrium rufum, $P$.

GENUS $X X I$.-STICTIS, $P$.

Sub-Genus 1.-EUSTICTIS. 1899. Stictis radiata, $P$.
1900. Stictis pallida, $\boldsymbol{P}$.

1901. Stictis microstoma, Carm.

1902. Stictis nivea, $P$.

1903. Stictis lichenicola, Mont.

1904. Stictis hysterioides, Desm.

1905. Stictis chrysophæa, $F r$.

Sub-Genus 2.-XYLOGRAPHA.

1906. Stictis parallela, Fr.

1907. Stictis longa, Fr.

Sub-Genus 3.-PROPOLIS.

1908. Stictis versicolor, $\mathrm{Fr}$.

1909. Stictis phacidioides, Fr.

1910. Stictis Wauchii, Grev.

GENUS XXII.-ASCOMYCES, Mont.

1911. Ascomyces bullatus, $B$.

1912. Ascomyces deformans, $B$.

1913. Ascomyces trientalis, $B$.

1914. Ascomyces juglandis, $B$.

DOUBTFUL OR EXTINCT SPECIES.

Leotia nana, Fr.

Peziza (Geopyxis) rapulum, Bull. Peziza (Geopyxis) argillacea, Sow. Peziza (Dasyscypha) Godroniana, Mont. - Vide P. tricolor, Sow., No. 1851. 


\section{Order XXIV.-TUBERACEI.}

GENUS I.-TUBER, Mich. ij15. Tuber brumale, Mich. 1916. Tuber æstivum, Vitt. 1917. Tuber macrosporum, Vitt. 1918. Tuber bituminatum, $B . \& B r$. 1919. Tuber rufum, Pico.

1920. Tuber scleroneuron, $B . \& B r$. 1921. Tuber nitidum, Vitt.

1922. Tuber puberulum, $B . \& B r$. 1923. Tuber dryophilum, Tul.

GENUS II.-CHOIROMYCES, Vitt.

1324. Choiromyces meandriformis, Vitt.

\section{GENUS III.-AMYLOCARPUS, Curr.}

1925. Amylocarpus encephaloides, Curr.

GENUS IV.-PACHYPHLGUS, Tul.

1926. Pachyphlœus melanoxanthus, Tul.

1927. Pachyphlœus citrinus,

B. \& Br.

1928. Pachyphlœus conglomeratus B. $\& B r$.
GENUS V.-STEPHENSIA, Tul. 1929. Stephensia bombycina, Tul.

GENUS VI.-HYDNOTRYA, $B . \& B r$.

1930. Hydnotrya Tulasnei, B.\& Br. GENUS VII.-HYDNOBOLITES Tul.

1931. Hydnobolites cerebriformis, Tul.

GENUS VIII.-SPH不OSOMA, $K l$.

1932. Sphærosoma ostiolatum, $T u l$. GENUS IX.-BALSAMIA, Vitt. 1933. Balsamia platyspora, B. \&Br.

GENUS X.-GENEA, Vitt. 1934. Genea verrucosa, Vitt. 1935. Genea Klotzschii, B. \& Br. 1936. Genea papillosa, Vitt. GENUS XI.-ELAPHOMYCES, Nees.

1937. Elaphomyces anthracinus, Vitt. 1938. Elaphomyces variegatus, Vitt. 1939. Elaphomyces granulatus, $F r$. 


\section{Order XXV.-PHACIDIACEI.}

GENUS I.-PHACIDIUM, Fr. 1940. Phacidium pini, Schm.

1941. Phacidium carbonaceum, $F r$.

1942. Phacidium vaccinii, $F r$.

1943. Phacidium coronatum, $F r$.

1944. Phacidium dentatum, $F r$.

1945. Phacidium rubi, $\mathrm{Fr}$.

1946. Phacidium repandum, $F r$.

1947. Phacidium simulatum, $B . \& B r$.

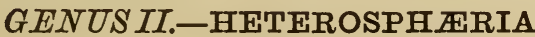
Grev.

1948. Heterosphæria patella, Grev.

GENUS III.-RHYTISMA, Fr.

1949. Rhytisma maximum, $\mathrm{Fr}$.

1950. Rhytisma andromedæ, $F r$.

1951. Rhytisma salicinum, $\mathbb{F r}$.

1952. Rhytisma acerinum, $F r$.

1953. Rhytisma punctatum, $F r$.

1954. Rhytisma urticæ, $F r$.

GENUS IV.-TRIBLIDIUM, Reb. 1955. Triblidium caliciiforme, $R e b$.

GENUS $V$.-HYSTERIUM, Tode. 1956. Hysterium pulicare, Pers.

1957. Hysterium elongatum, Wahl.

1958. Hysterium curvatum, $F r$.

1959. Hysterium lineare, $F r$.

1960. Hysterium Carmichaelianum

$B$.
1961. Hysterium fraxini, $\boldsymbol{P}$. 1962. Hysterium conigenum, ILoug. \& $N$. 1963. Hysterium vaccinii, Carm. 1964. Hysterium rubi, Pers. 1965. Hysterium pinastri, Schrad. 1966. Hysterium melaleucum, $F r$. 1967. Hysterium commune, $F r$. 1968. Hysterium typhinum, $F r$. 1969. Hysterium arundinaceum, Schrad.

1970. Hysterium culmigenum, $\mathrm{Fr}$. 1971. Hysterium maculare, $F r$. 1972. Hysterium foliicola, $F r$. GENUS VI.-AILOGRAPHUM, 1973. Ailographum amplum, B. $\& B r$.

1974. Ailographum maculare, B. \&Br.

GENUS VII.-ASTERINA, Lev. 1975. Asterina Babingtonii, $B$.

GENUS VIII.-LOPHIUM, Fr. 1976. Lophium elatum, Grev. 1977. Lophium mytilinum, $F r$.

GENUS IX.-STEGIA, Fr. 1978. Stegia ilicis, $F r$.

GENUS X.-TROCHILA, Fr. 1979. Trochila craterium, $\mathrm{Fr}$. 1980. Trochila lauro-cerasi, $F r$. 


\section{Order XXVI.-SPHÆRIACEI.}

GENUS I-CORDICEPS, Fr. 1981. Cordiceps militaris, $\mathrm{Fr}$. 1982. Cordiceps entomorrhiza, $F r$. 1983. Cordiceps capitata, $\mathrm{Fr}$. 1984. Cordiceps ophioglossoides, $\operatorname{Fr}$ 1985. Cordiceps gracilis, $B$. 1986. Cordiceps purpurea, $F r$. 1987. Cordiceps microcephala, $T u l$. 1988. Cordiceps myrmecophila, $B$. 1989. Cordiceps alutacea, $F r$. 1990. Cordiceps pistillariæformis, B. \& Br.

GENUS II.-HYPOCREA, Er. 1991. Hypocrea gelatinosa, $\mathrm{Fr}$. 1992. Hуроcrea rufa, $F r$. 1993. Hypocrea riccioidea, $B$. 1994. Hypocrea vitalba, $B . \& B r$. 1995. Hypocrea citrina, $F r$. 1996. Нуросrea lateritia, $F r$. 1997. Hуросrea luteo-virens, $F r$. 1998. Hypocrea farinosa, $B . \& B r$. 1999. Нypocrea floccosa, $F r$. 2000. Hypocrea typhina, $B$. 2001. Hypocrea inclusa, $B . \& B r$. GENUS III.-ENDOTHIA, $F r$. 2002. Endothia gyrosa, $F r$.

GENUS IV.-XYLARIA, Schr. 2003. Xylaria polymorpha, Grev.
2004. Xylaria digitata, Grev. 2005. Xylaria cớrniformis, Mont. 2006. Xylaria hypoxylon, Grev. 2007. Xylaria carpophila, $F r$. 2008. Xylaria pedunculata, $F r$. 2009. Xylaria bulbosa, $B$. \& Br. 2010. Xylaria vaporaria, Berk.

\section{GENUS V.-THAMNOMYCES, Ehrb.}

2011. Thamnomyces hippotrichioides, Ehrb.

GENUS VI.-PORONIA, Fr. 2012. Poronia punctata, Fr.

$$
\text { GENUS VII.-HYPOXYLON, }
$$

2013. Hypoxylon ustulatum, Bull. 2014. Hypoxylon nummularium, Bull.

2015. Hypoxylon luteum, $F r$.

2016. Hypoxylon succenturiatum, Fr.

2017. Hypoxylon gastrinum, Fr. 2018. Hypoxylon concentricum, Grev. 2019. Hypoxylon coccineum, Bull. 2020. Hypoxylon multiforme, $F r$. 2021. Hypoxylon marginatum, $B$. 2022. Hypoxylon cohærens, $F r$. 2023. Hypoxylon argillaceum, $F r$. 2024 Hypoxylon fuscum, Fr. 
2025. Hурoxylon rubiginosum, Fr. 2026. Hypoxylon atropurpureum, Fr.

2027. Hypoxylon serpens, Fr. 2028. Hypoxylon coprophilum, Fr. 2029. Нурохylon udum, Fr.

GENUS VIII.-DIATRYPE, Fr. a Lignosce.

2030. Diatrype bullata, $F r$. 2031. Diatrype undulata, Fr. 2032. Diatrype stigma, Fr. 2033. Diatrype disciformis, Fr. 2034. Diatrype aspera, Fr. 2035. Diatrype favacea, $F r$. 2036. Diatrype verrucæformis, $F r$. 2037. Diatrype lanciformis, $F r$. 2038. Diatrype quercina, Fr. 2039. Diatrype dryophila, Curr. 2040. Diatrype nucleata, Curr. 2041. Diatrype varians, Curr. 2042. Diatrype denigrans, Curr. 2043. Diatrype Badhami, Curr. 2044. Diatrype inæqualis, Curr.

\section{$\beta$ Versatiles.}

2045. Diatrype scabrosa, $F r$. 2046. Diatrype ulicis, $B$. 2047. Diatrype podoides, $F r$. 2048. Diatrype ferruginea, Fr. 2049. Diatrype flavo-virens, $F r$. 2049A. Diatrype flavo-virens, var. $\beta$ multiceps, Sow.

2050. Diatrype sordida, $B . \& B r$. 2051. Diatrype hystrix, $F r$. 2052. Diatrype ceratosperma, Fr.
2053. Diatrype strumella, Fr.

2054. Diatrype pyrrhocystis, B. \& Br.

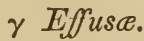

2055. Diatrype incarcerata, B. \& Br.

2056. Diatrype stipata, Curr.

2057. Diatrype elevata, $B . \& B r$.

2058. Diatrype leioplaca, Fr.

2059. Diatrype lata, Fr.

2060. Diatrype decipiens, Fr.

$\delta$ Circumscriptce.

2061. Diatrype corniculata, B. \& Br.

2062. Diatrype cincta, $B . \& B r$.

GENUS IX.-VALSA, Fr.

a Circumscripta.

2063. Valsa prunastri, Fr.

2064. Valsa stellulata, Fr.

2065. Valsa enteroleuca, Fr.

2066. Valsa extensa, Fr.

2067. Valsa syngenesia, $F r$.

2068. Valsa cratægi, Curr.

2069. Valsa detrusa, $\mathrm{Fr}$.

2070. Valsa fibrosa, Fr.

$\beta$ Incusce.

2071. Valsa nivea, $F r$.

2072. Valsa leucostoma, $F r$.

2073. Valsa Kunzei, Fr.

2074. Valsa angulata, $F r$.

2075. Valsa luteola, $F r$.

2076. Valsa microstoma, Fr.

2077. Valsa profusa, $F r$.

2078. Valsa dissepta, Fr.

2079. Valsa controversa, Fr. 
20s0. Valsa dryina, Curr.

2081. Valsa concamerata, Curr.

$\gamma$ Obvallata.

2082. Valsa coronata, Fr.

2083. Valsa abietis, Fr.

2084. Valsa chrysostroma, Fr.

2085. Valsa suffusa, Fr.

2086. Valsa leiphæmia, $F r$.

2087. Valsa turgida, $\mathrm{Fr}$.

2088. Valsa salicina, $F r$.

2089. Valsa ambiens, Fr.

2090. Valsa stilbostoma, $F r$.

2091. Valsa platanoides, $B$.

2092. Valsa platanigera, $B . \& B r$.

2093. Valsa tetratrupha, $B . \& B r$.

2094. Valsa fenestrata, $B . \& B r$.

2095. Valsa tetraploa, B. \& Curt.

2096. Valsa rhodophila, $B . \& B r$.

2097. Valsa quernea, Curr.

2098. Valsa biconica, Curr.

2099. Valsa pulchra, Curr.

2100. Valsa tetraspora, Curr.

2101. Valsa intexta, Curr.

$\delta$ Circinate.

2102. Valsa pulchella, $F r$.

2103. Valsa quaternata, Fr.

2104. Valsa furfuracea, $F r$.

2105. Valsa hypodermia, Fr.

2106. Valsa convergens, Fr.

2107. Valsa hapalocystis, $B . \& B r$.

2108. Valsa bitorulosa, $B . \& B r$.

2109. Valsa aglæostoma, $B . \& B r$.

2110. Valsa Innesii, Curr.

2111. Valsa faginea, Curr.

2112. Valsa tilaginea, Curr.
2113. Valsa vestita, Fr.

2114. Valsa lageniformis, Curr.

GENUS X.-MELOGRAMMA, Fr.

2115. Melogramma rubro-notatum B. $\& B r$.

2116. Melogramma oligosporum, B. $\& . B r$.

2117. Melogramma fusisporum, Fr.

2118. Melogramma homaleum, Fr. GENUS XI.-DOTHIDEA, Fr.

2119. Dothidea tetraspora, B. \& Br.

2120. Dothidea ribesia, Pers.

2121. Dothidea rosæ, Fr.

2122. Dothidea Piggotii, B.\& $B r$.

2123. Dothidea filicina, $F r$.

2124. Dothidea striæformis, $F r$.

2125. Dothidea rubra, Pers.

2126. Dothidea fulva, Holl. \& Schm.

2127. Dothidea ulmi, Fr.

2128. Dothidea betulina, $F r$.

2129. Dothidea heraclei, $\mathrm{Fr}$.

2130. Dothidea podagrariæ, Fr.

2131. Dothidea trifolii, Fr.

2132. Dothidea junci, $F r$.

2133. Dothidea graminis, $F r$.

2134. Dothidea caricis, Fr.

2135. Dothidea Johnstoni, B. \&Br .

GENUS XII.-ISOTHEA, Fr. 2136. Isothea rhytismoides, $F r$.

2137. Isothea pustula, $B$.

2138. Isothea saligna, $B$.

GENUS XIII.-HYPOSPILA, F'r. 2139. Hypospila quercina, Fr. 
2140. Hypospila populina, $F r$.

GENUS XIV.-STIGMATEA, Fr.

2141. Stigmatea conferta, $F r$.

2142. Stigmatea geranii, Fr.

2143. Stigmatea ranunculi, Fr.

2144. Stigmatea Robertiani, Fr.

2145. Stigmatea polygonorum, $F r$.

2146. Stigmatea alchemillæ, Grev.

2147. Stigmatea chætomium, $F r$.

GENUS $X V$-OOOMYCES, B. $\& B r$.

2148. Oomyces carneo-albus, B. \& Br.

\section{GENUS XVI.-NECTRIA, $F r$.}

a Caspitosa.

2149. Nectria ochracea, Fr.

2150. Nectria cinnabarina, $F r$.

2151. Nectria coccinea, Fr.

2152. Nectria cucurbitula, Fr.

2153. Nectria sinopica, Fr.

2154. Nectria aquifolia, $B$.

2155. Nectria inaurata, $B . \& B r$.

2156. Nectria Ralfsii, $B . \& B r$.

2157. Nectria hirta, Blox.

$\beta$ Byssiseda.

2158. Nectria aurantia, $F r$.

2159. Nectria rosella, Fr.

2160. Nectria Albertini, $B . \& B r$.

y Villose.

2161. Nectria flavida, $F r$.

2162. Nectria funicola, B.\& $\mathrm{Br}$.

\section{$\delta$ Denudata.}

2163. Nectria peziza, $F r$.

2164. Nectria platasca, $B$.
2165. Nectria sanguinea, $F r$.

2166. Nectria episphæria, Fr.

2167. Nectria Purtoni, Curr.

2168. Nectria ochraceo-pallida, B. \& Br.

2169. Nectria muscivora, $B . \& B r$.

2170. Nectria arenula, $B . \& B r$.

2171. Nectria graminicola, B. \& $B r$.

2172. Nectria Bloxami, B. \& Br.

2173. Nectria helminthicola, B. \& Br.

2174. Nectria Rousseliana, Mont.

2174A. Nectria Rousseliana, var $b$. viridis, $B$.

2174в. Nectria Rousseliana, var. c. fulva, $B$.

2175. Nectria umbrina, Fr.

\section{GENUS XVII.- SPH FRIA, Hall.}

\section{A.-Superficiales.}

$a$-BYSSISED $\mathbb{E}$.

2176. Sphæria thelena, $\mathrm{Fr}$.

2177. Sphæria aquila, Fr.

2178. Sphæria Desmazierii, B. $\& B r$.

2179. Sphæria Dickiei, $B . \& B r$.

2180. Sphæria tristis, Tode.

2181. Sphæria phæostroma, Mont.

2182. Sphæria biformis, $P$.

218:3. Sphæria racodium, $P$.

b-VILLOSA.

2184. Sphæria ovina, $P$.

2185. Sphæria cæsia, Curr.

2186. Sphæria mutabilis, $P$. 
2187. Sphæria brassicæ, Klot.

2188. Sphæria scabra, Curr.

2189. Sphæria canescens, $P$.

2190. Sphæria strigosa, $A . \& S$.

2191. Sphæria hirsuta, Fr.

2192. Sphæria callimorpha, Mont.

2103. Sphæria macrotricha, B. \& $B r$

2194. Sphæria chætomium, $C d$. 2195. Sphæria eres, $B$. \& Br. 2196. Sphæria exilis, $A$. \& $S$. 2197. Sphæria calva, Tode. 2198. Sphæria pilosa, $P$. 2199. Sphæria hispida, Tode. 2200. Sphæria pellita, $F r$. 2201. Sphæria superficialis, Curr. 2202. Sphæria capillifera, Curr. 2203. Sphæria scatigena, $B . \& B r$. c-DENUDATA.

2204. Sphæria bombarda, Batsch. 2205. Sphæria spermoides, Hoffm. 2206. Sphæria papaverea, $B$. \& $B r$. 2207. Sphæria moriformis, Tode. 2208. Sphæria innumera, $B . \& B r$. 2209. Sphæria confluens, Tode. 2210. Sphæria botryosa, Fr. 2211. Sphæria stercoraria, Scy. 2212. Sphæria mammæformis, $P$. 2213. Sphæria pomiformis, $P$. 2214. Sphæria sordaria, $F r$. 2215. Sphæria obducens, $F r$. 2216. Sphæria pulvis-pyrius, $P$. 2217. Sphæria rhytidodes, $B . \& B r$. 2218. Sphæria perexigua,
2219. Sphæria pulveracea, Ehr. 2220. Sphæria ordinata, $F r$. 2221. Sphæria moroides, Curr. 2222. Sphæria myriocarpa, $F r$. 2223. Sphæria vesticola, $B . \& B r$. 2224. Sphæria rubicola, Curr. 2225. Sphæria collabens, Curr. 2226. Sphæria caudata, Curr. 2227. Sphæria Curreyii, Blox. 2228. Sphæria pulviscula, Curr. d-PERTUSA.

2229. Sphæria pertusa, $P$. 2230. Sphæria callicarpa, Curr. 2231. Sphæria Aspegrenii, $F r$. 2232. Sphæria Jenynsii, $B . \& B r$. 2233. Sphæria pœcilostoma, B. \&Br.

2234. Sphæria brachythele, B. \&Br.

2235. Sphæria vilis, $F r$.

2236. Sphæria mastoidea, Fr. 2237. Sphæria nucula, Fr.

\section{3.-ERUMPentes. e-CASPITOS死.}

2238. Sphæria populina, $P$. 2239. Sphæria cupularis, $P$. 2240. Sphæria acervata, $F r$. 2241. Sphæria dioica, Moug. 2242. Sphæria berberidis, $P$. 2243. Sphæria laburni, $P$. 2244. Sphæria nigerrima, Blox. 2245. Sphæria barbula, $B . \& B r$. 2246. Sphæria apotheciorum, Mass.

2247. Sphæria nidula, Sow. 
2248. Sphæria naucosa, $F r$.

$$
f \text {-OBTURATA. }
$$

2249. Sphæria clongata, $F r$.

2250. Sphæria spartii, $B$.

2251. Sphæria scoriadea, $F r$.

2252. Sphæria juglandis, $F r$.

2253. Sphæria loniceræ, Sow.

2254. Sphæria obliterans, $B . \& B r$.

2255. Sphæria Godini, Desm.

2256. Sphæria culmifraga, $\mathrm{Fr}$.

g-LOPHIOSTOMÆ.

2257. Sphæria excipuliformis, $F r$.

2258. Sphæria angustilabra,

$$
\text { B. } \& B r \text {. }
$$

2259. Sphæria macrostoma, Tode.

2260. Sphæria fibritecta, $B$.

2261. Sphæria arundinis, $F r$.

\section{$h$-CERATOSTOM}

2262. Sphæria cirrhosa, $P$.

2263. Sphæria pilifera, $F r$.

2264. Sphæria ligneola, $B . \& B r$.

2265. Sphæria lampadophora, $B . \& B r$.

2266. Sphæria stylophora, $B . \& B r$.

$$
\text { C.-SUвteCtz. }
$$

2267. Sphæria spinosa, $P$. 2268. Sphæria eutypa, $F r$. 2269. Sphæria livida, $F r$. 2270. Sphæria melanotes, $B . \& B r$. 2271. Sphæria hypotephra, B. $\& B r$. 2272. Sphæria hemitapha, $B . \& B r$. 2273. Sphæria apiculata, Curr. 2274. Sphæria anserina, $P$.
* * Endophloce.

2275. Sphæria velata, $P$. 2276. Sphæria ciliaris, Curr. 2277. Sphæria cælata, Curr. 2278. Sphæria xylostei, $P$. 2279. Sphæria decedens, $F r$. 2280. Sphæria discutiens, $B$. 2281. Sphæria fraxinicola, Curr. 2282. Sphæria verecunda, Curr.

*** Endocaula.

2283. Sphæria spiculosa, $P$. 2284. Sphæria inquilina, $F r$. 2285. Sphæria Berkeleii, Desm. 2286. Sphæria scirpicola, $D C$. 2287. Sphæria phomatospora, B. \&Br.

2288. Sphæria blepharodes, B. \& Br.

$$
\begin{gathered}
\text { j-OBTECT } \mathrm{E} . \\
\text { * Rameales. }
\end{gathered}
$$

2289. Sphæria obtecta, Curr. 2290. Sphæria lanata, $F r$. 2291. Sphæria siparia, $B . \& B r$. 2292. Sphæria holoschista, B. $\& B r$.

2293. Sphæria Glis, B. \& Curr. 2294. Sphæria pruinosa, $F r$. 2295. Sphæria unicaudata, $B . \& B r$. 2296. Sphæria vibratilis, $F r$. 2297. Sphæria millepunctata, Grev. 2298. Sphæria salicella, Fr. 2299. Sphæria Argus, $B . \& B r$. 2300. Sphæria gigaspora, Desm. 2301. Sphæria corni, Mont. 2302. Sphæria aucta, $B . \& B r$. 2303. Sphæria bufonia, $B . \& B r$. 
2304. Sphæria dochmia, $B . \& B r$. 2305. Sphæria farcta, $B . \& B r$. 2306. Sphæria trivialis, $B . \& B r$. 2307. Sphæria revelata, $B . \& B r$. 2308. Sphæria quadrinucleata, Curr. 2309. Sphæria conformis, $B . \& B r$. 2310. Sphæria rubi, Curr.

2311. Sphæria fuscella, $B . \& B r$. 2312. Sphæria sepincola, $F r$. 2313. Sphæria persistens, $B . \& B r$. 2314. Sphæria futilis, $B . \& B r$. 2315. Sphæria intermixta, $B . \& B r$. 2316. Sphæria oblitescens, $B . \& B r$. 2317. Sphæria epidermidis, $F r$. 2318. Sphæria tamaricis, Grev. 2319. Sphæria ocellata, $F r$. 2320. Sphæria melina, $B . \& B r$. 2321. Sphæria Ashwelliana, Curr. 2322. Sphæria clypeata, Nees. 2323. Sphæria appendiculosa, B. \& $B r$.

2324. Sphæria pusilla, Curr. 2325. Sphæria rusci, Wallr. 2326. Sphæria pinastri, $F r$.

\section{* * Herbicola.}

2327. Sphæria tomicum, Lév. 2328. Sphæria herpotricha, $F r$. 2329. Sphæria lirella, $P$. 2330. Sphæria acus, Blox. 2331. Sphæria lineolata, $R o b$. 2332. Sphæria maculans, Sow. 2333. Sphæria pantherina, $B$. 2334. Sphæria pardalota, Mont. 2335. Sphæria ceuthosporoides, $B$.
$k$-CAULICOL无.

2336. Sphæria curvirostra, Sow. 2337. Sphæria rostellata, $F r$. 2338. Sphæria coniformis, $F r$. 2339. Sphæria acuminata, Sow. 2340. Sphæria cruciferarum, Desm. 2341. Sphæria herbarum, $P$. 2342. Sphæria rubella, $P$. 2343. Sphæria Helenæ, Curr. 2344. Sphæria doliolum, $P$. 2345. Sphæria complanata, Tode. 2346. Sphæria nigrella, $F r$. 2347. Sphæria corni-suecicæ, $F r$. 2348. Sphæria derasa, $B . \& B r$. 2349. Sphæria tritorulosa, B. \& $\mathrm{Dr}$. 2350. Sphæria vectis, $B . \& B r$. 2351. Sphæria planiuscula, B. \& Br. 2352. Sphæria lunariæ, $B . \& B r$. 2353. Sphæria nigrans, Desm. 2354. Sphæria semilibera, Desm. 2355. Sphæria Ogilviensis, B. \& Br. 2356. Sphæria Clivensis, B. \& Br. 2357. Sphæria modesta, Desm. 2358. Sphæria commanipula, B. $\& B r$. 2359. Sphæria Thwaitesii, $B . \& B r$. 2360. Sphæria tosta, $B . \& B r$. 2361. Sphæria tenebrosa, B. \& Br . 2362. Sphæria glœospora, B. \& Curr. 2363. Sphæria pinodes, B. \& Blox. 2364. Sphæria caulium, Fr. 2365. Sphæria triglochinicola, Curr. 


\section{l一FOLIICOLA. \\ * Rostellatce.}

2366. Sphæria fimbriata, $B$. 2367. Sphæria coryli, Batsch. 2368. Sphæria avellanæ, Schm. 2369. Sphæria tubæformis, Tode. 2370. Sphæria gnomon, Tode. 2371. Sphæria setacea, $P$. 2372. Sphæria cariceti, $B . \& B r$. 2373. Sphæria microspila, $B . \& B r$. ** Sphcrostomce.

2374. Sphæria duplex, Sow. 2375. Sphæria recutita, $\dot{F r}$. 2376. Sphæria anarithma, $B . \& B r$. 2377. Sphæria sabuletorum, B. $\& B r$.

2378. Sphæria phæosticta, $B$.

2379. Sphæria eucrypta, $B . \& B r$. 2380. Sphæria helicospora, B.\& Br . 2381. Sphæria palustris, $B$. \& $B r$. 2382. Sphæria carpinea, Fr. 2383. Sphæria pteridis, Desm. 2384. Sphæria brassicæcola, B. $\& B r$.

2385. Sphæria punctiformis, $F r$. 2386. Sphæria maculæformis, $P$. 2387. Sphæria eryngii, $F r$. 2388. Sphæria rumicis, Desm. 2389. Sphæria Leightoni, $B . \& B r$. 2390. Sphæria erysiphina, $B . \& B r$. 2391. Sphæria brunneola, $F r$. 2392. Sphæria ostruthii, $F r$. 2393. Sphæria ligustri, Desm.
GENUS XVII.-CERATOSTOMA, $F r$.

2394. Ceratostoma caprinum, Fr. 2395. Ceratostoma Zobelii, $B$.

GENUS $X V I I I$ - MASSARIA, De Not.

2396. Massaria fœdans, $F r$. 2397. Massaria inquinans, $F r$. 2398. Massaria fimeti, $F r$.

GENUS XIX.-HERCOSPORA, Fr.

2399. Hercospora pupula, Fr.

GENUS $X X$.-PYRENOPHORA, Fr.

3000. Pyrenophora phæocomes, Fr.

GENUS $X X I$.-GIBBERA, $F r$. 3001. Gibbera vaccinii, $F r$. 3002. Gibbera pulicaris, $F r$. 3003. Gibbera Saubinetii, Mont. GENUS XXII.-DICH正NA, Fr. 3004. Dichæna rugosa, Fr. 3005. Dichæna strobilina, Fr. GENUS XXIII-CAPNODIUM, Mont.

3006. Capnodium elongatum, B. \& Desm.

DOUBTFUL OR EXTINCT SPECIES.

Sphæria fuliginosa, $F r$. 


\section{ORDER XXVII._PERISPORIACEI.}

GENUS I.-THELEBOLUS, Tode.

3007. Thelebolus terrestris, $A . \& S$. GENUS II.-PERISPORIUM, $F r$. 3008. Perisporium princeps, $B$. 3009. Perisporium arundinis, $F r$. GENUS III.-LASIOBOTRYS, 3010. Lasiobotrys loniceræ, Kze. 3011. Lasiobotrys Linneæ, $B$.

\section{GENUS IV.-SPH正ROTHECA,} Lev.

3012. Sphærotheca pannosa, Lev. 3013. Sphærotheca Castagnei, Lev.

$$
\text { GENUS V.-PHYLLACTINIA, }
$$

3014. Phyllactinia guttata, $F r$.

GENUS VI._UNCINULA, Lev. 3015. Uncinula adunca, Lev. 3016. Uncinula bicornis, Lev.

\section{GENUS VII.-MICROSPH开-} RIA, Lev.

3017. Microsphæria berberidis,
3018. Microsphæria grossulariæ, Lev.

3019. Microsphæria penicillata, Lev.

GENUS VIII.-ERYSIPHE, Hedw.

3020. Erysiphe Linkii, Lev. 3021. Erysiphe lamprocarpa, $L k$. 3022. Erysiphe graminis, $D C$. 3023. Erysiphe Martii, $B$. 3024. Erysiphe Montagnei, Lev. 3025. Erysiphe tortilis, $L k$. 3026. Erysiphe communis, Schl.

\section{GENUS IX.-CH出TOMIUM, Kze.}

3027. Chætomium elatum, $K z e$. 3028. Chætomium chartarum, Ehrb. 3029. Chætomium glabrum, $B$. GENUS X.-ASCOTRICHA, $B$. 3030. Ascotricha chartarum, $B$. GENUS XI.-EUROTIUM, Lk. Lev. 3031. Eurotium herbariorum, $L k$.

\section{ORDER XXVIII.-ONYGENEI.}

GENUS I;-ONYGENA, $P$. $\mid$ 3033. Onygena piligena, $F r$. 3032. Onygena equina, Pers. 3034. Onygena apus, B. \&Br. 


\section{FAMILY VI.-PHYSOMYCETES.}

\section{ORDER XXIX.-ANTENNARIEI.}

\begin{tabular}{r|c} 
GENUS I.-ANTENNARIA, Lk. & GENUS II.-ZASMIDIUM, \\
3035. Antennaria semiovata, & Fr. \\
B. \& Br. & 3036. Zasmidium cellare, Fr.
\end{tabular}

\section{ORdER XXX.-MUCORINI.}

GENUS I.-ASCOPHIORA, Tode. 3037. Ascophora mucedo, Tode. 3038. Ascophora elegans, $C d$.

- GENUS II.-MUCOR, Mich. 3039. Mucor phycomyces, Berk. 3040. Mucor ramosus, Bull. 3041. Mucor mucedo, $L$. 3042. Mucor caninus, $P$. 3043. Mucor fusiger, $L k$. 3044. Mucor clavatus, $L k$. 3045. Mucor amethysteus, Berk. 3016. Mucor succosus, Berk. 3047. Mucor delicatulus, Berk. 3048. Mucor tenerrimus, Berk. 3049. Mucor subtilissimus, Berk. GENUS III.-PILOBOLUS, Tode. 3050. Pilobolus roridus, Bolt. 3051. Pilobolus crystallinus, Tode. GENUS IV.-HYDROPHORA,
Tode.

3052. Hydrophora stercorea, Tode.

GENUS V.-ENDODROMIA, Berk.

3053. Endodromia vitrea, $B$.

GENUS VI.-SPORODINIA, $L k$. 3054. Sporodinia dichotoma, $C d$.

GENUS VII.-ACROSTALAGMUS, $\mathrm{Cd}$.

3055. Acrostalagmus cinnabarinus,

$c d$.

GENUS VIII.-SYZYGITES, Ehb.

3056. Syzygites megalocarpus, Ehb.

GENUS IX.-ENDOGOINE, $L k$. 3057. Endogone pisiformis, $L k$. 3058. Endogone lactiflua, B. \& Br. 


\section{INCERTA SEDIS.}

GENUS I.-SCLEROTIUM, Tode. 3059. Sclerotium complanatum, 3060. Sclerotium scutellatum, Tode. A. \& S.

3061. Sclerotium semen, Tode.

3062. Sclerotium cepævorum, Berk.

3063. Sclerotium stercorarium, $D C$.

3064. Sclerotium quercigenum, Berk.

3065. Sclerotium truncorum, Tode. 3066. Sclerotium fungorum, $P$. 3067. Sclerotium lacunosum, $P$. 3068. Sclerotium muscorum, $P$.
3069. Sclerotium roseum, Kneiff. 3070. Sclerotium varium, $P$. 3071. Sclerotium medullare, Berk. 3072. Sclerotium neglectum, Berk. 3073. Sclerotium pyrinum, Fr. 3074. Sclerotium rubi, Carm. 3075. Sclerotium bullatum, $D C$. 3076. Sclerotium durum, $P$. 3077. Sclerotium pustula, $D C$.

GENUS II.-RHIZOCTONIA, $1 \quad D C$.

3078. Rhizoctonia crocorum, $D C$. 3079. Rhizoctonia menthæ, Berk.

\section{E R R A T U M.}

\section{Page 56, No. 3011. Lasiobotrys Linneæ, $B$.}

This Species, as far as I can ascertain, is identical with No. 2179 Sphæria Dickiei, B. \& Br., Venturia Dickiei, Ces. \&De Not. in "Schema di Classificazione degli Sferiacei Italici," Genoa, 1863. 


\title{
JOURNAL OF BOTANY, BRITISH AND FOREIGN.
}

\author{
EDITED BY \\ BERTHOLD SEEMANN, Ph.D., F.L.S., F.R.G.S.
}

\section{PAPERS ON BRITISH AND CRYPTOGAMIC BOTANY}

Will pass through the hands of those who have specially attended to these subjects.

THe fact that at present, when almost every braneh of human inquiry has its special $\mathrm{Pe}$ riodical, there is not in the whole of Great Britain a Magazine exclusively devoted to the science of Botany in all its bearings, in which those who have not the advantage of attending the Meetings of Learned Societies are able to exchange their opinions, has induced the Editor to project the 'Journal of Botany, British and Foreign.'

The Journal consists of two distinct sections : the one devoted to General Botany, the other to the Botany of the British Isles. In both divisions Original Papers on subjects new to Science are given, and when necessary, illustrated either in the text or by special Plates. Each numbcr contains a full-page Plate by Fitch, illustrative of New Species described in the letter-press. Original Communications of necessity rclate to the different sections of the Science, and form the bulk; yet Structural, Morphological, and Physiolugical Botany are attended to. Nor is Geographical nor Palæontological Botany neglected. The Editor specially watches over this, the important portion of the Journal, and he confidently hopes that the excellence of these communications will make this Journal a Standard Work, and obtain for it a circulation where otherwise it would be unknown. Mere details of Excursions, either in well-known or little-known countries, with records of collecting already known plants, are excluded, unless there is evidently some real addition to knowledge contained in them. By exercising a careful revision of everything inserted in the Journal, and by employing every portion of it in publishing real and solid information, the Editor fears not that he will receive the support of Botanists generally. That he does not promise more than he can accomplish must be evident, when we reflect that all he proposes is the publication, in Great Britain, of one monthly Journal devoted to Botany,--a science so extensive, and making at present such rapid strides, that the difficulty to the Editor will often be what to exclude, rather than how to fill up the few monthly sheets. Special attention will be paid to Botanical News. The large number of Continental Botanical Societies and Periodicals are almost unknown in this country. The readers of the Journal will be supplied with a careful and speedy digest of these publica. tions. Bibliography will also receive attention, not only in Reviews, but in Systcmatic Lists, accompanied with short Critical Notices.

\section{CONTENTS OF THE TWELVE PARTS, FORMING THE FIRST VOLUME.}

\section{PART I.}

On British Species of Isoëtes. By C. C. Babington, M.A., F.R.S.-Anthurium gladiifolium, a new Brazilian Aroidea. By Dr. H Schott. - On Certain Varietıes in the Common Rye-Grass. By Maxwell T. Masters, -M.D., F.L.S.-On Tecophileacea, a New Natural Order of Monocotyledonous Plants. By Dr. F. Leybold.-On some of the British Pansies, agrestal and montane. By J. G. Baker, Esq.-On Tryblionella Victorice and Denticula subtilis, two Species of British Diatomacex. By W. Carruthers, Esq., F.L.S.-Kevision of the Natural Order Bignoniacece. By B. Seemann, Ph.D., F.L S.-Menıranda : The Sago-Palms of the Aru Islands; New British Algr ; Common I.ing; Arum Canariense.New Publications: Benthain's 'Handbook of the British Flora'; Syme's Edition of 'Sowerby's English Botany.'-Botanical News.

\section{PART II.}

Podocarpus Vitiensis, a new Coniferous Tree, from the Viti Islands. By B. Seemann. (With a Plate.)
Chinchona Cultivation in India. Communicated by C. R. Markhain, F.S.A., F.R.G.S.-Iypnum exannulatum, a New British Moss. By W. Carruthers. -Cork-tree at Summertown, near Cork, Ireland. By C. C. Babington.-Sturmia Loeselii. By C. C. Babington.-The unusually Mild Winter.-New Publications : Gibson's 'Flora of Essex '; The Transactions of the Linnean Society of London, Vol. XXIV., Part I.-Botanical News.

\section{PART III.}

Rare or New British Hymenomycetal Fungi $B y$ M. C. Cooke.-Opening of Palm Spathest with an Audible Report. By Alexander Smith.-On the Tertiary Flora of the Aretic Region. By Prof. H. R. Goeppert.-Revision of the Natural Order Bignoniacea. By B. Seemann.-Note on Floræ Sarnicæ.-Correspondence: On Tecophileacere, a New Natural Order of Monocotyledonous Plants. By Jolın Miers, F.R.S. ; Popular. Names of British Plants. By Dr. Prior, F.L.S.-New Publications: Moore's 'Index Filicum'; More's 'Comparative List of British Plants.-Botanical News. 


\section{P'AR'T IV.}

On Cilaciolus Illyrious, as a British Plant. By C. C. Babington. (With a I'late.)-Was the CocoaNut known to the Ancimt ligyptians? By 13 . Seemann.-Galinsoga parvifora, Cav., a Natnralizcel British Plant. On the Morploology and Anatomy of I'hilydrum lanuginosum, I3r.-On the A natomy of

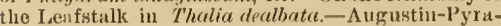
uns De Candolle. By Asa Gray.-Memoranda: Liquor Prepared from the Cassava Root; Colouring Matter of the Rerl Sea--New Publications: Scliubeler's 'Culturpflanze' Norwegens.'-Botan. News.

\section{I'AR'T V.}

Tropaolum IIeyneanum, a little-known specics from Southern Peru. By B Scemann. (With $a$ Plate.)-Remarks on Gladiolus Illyricus, Koeh., and its Allies. By J.'T. Boswell Syme, F.L.S - On a New Cliaracter in the Fruit of Quercus, and on the Best Sulodivision of that Genus. By Alphonse de Candolle - Report for 1862 of the Thirsk Botanical Exchange Club. By J. G. Baker.-1)imorphisin in the Genitalia of Flowers. IBy Asa Gray.-Correspondence: Vegetation about Cape Arid, Soutlwest Australia; Explosion of the Pods of Acanthus mollis; Opening of Palm Spathes with an Audible Report; Popular Names of British Plants; Dr. Nylander's Criticisms on Mudd's 'Herbarium Lidenum Britannicorum.' - New Publications : I3aker's 'North Yorkslire.'-Botanical News.

\section{PART VI.}

On the Nardoo Plant of Australia. By F. Currey, M.A., F.R.S. (With a Plate.)-On-the Arrangement of the British Saliees. By C. C. Babington.-Distinctive Charaeters of Quercus and Castanea, with Remarks on some Genera of Corylaceæ. Ify H. F. Hance, Ph.D., etc.-Asplenium Serpentini, Tausch, a Recent Addition to the British Ferns.-Carpomitra Cabrera on the Jersey Coast.-Viviparous Reproduetion of Sagina nodosa.-Fecundation of Gloxinia precta.-Memorandum : Edible Plants of Port Lincoln, Australia. - New Publications: Balfour's 'Flora of Edinburgh'; Holle's 'Flora von Hannover ;' Holle's 'Farnflor'a der Gegend von Hannover '; s'quier's 'Tropical Fibres.'-Botanical News.

\section{PART VII.}

On Chara alopecuroides, Del., as a Native of Britain. By C. C. Babington. (With a Plate.)-A Bipinnate Cycadea from N.E. Australia. By B. Seemann.-Contribution to the History of Aroideology. By H. W. Schott.-The Solana of Tropieal Polynesia. By B. Seemann.-Report to the Under-Secretary of State for India on the Bark and Leaves of Chinchona succirubra grown in India. By J. E. Howard, F.L.S.-Correspondence: Qumine, Shinehonidine, and Chinchonine, in the Leaves of Chinchona succirubra; Bryologieal Notes; 'The new Purple Trefoil of the Selly Islands.-New Publicatıons: Benthan's 'Flora Australiensis '; Rabenhorst's 'Cryptogamic Flora of Saxony, Upper Lusatia, Thuringia, and Northern Bohemia '; Crepin's L'Ardenne. - Botanical News.

\section{VIII.}

Revision of the Natural Order Bignoniaceæ. By B. Seemann. (With a Plate.)-Hypnum exannulatum. Br. \& Sch., and H. aduncum, L. By W. Carruthers. -Adnotationes in Cassiniaceas Wrightianas $\mathrm{Cu}$ benses, a $\mathrm{Cl}$. Grisebach determinatas. By C. H. Schultz-Bipontinus.-Trichomanes radicans indigenous to Yorkshire and Wales. By'Thomas Moore, F.L.S.-The Ordeal Bean of Calabar (Physostigma venenosum, Balf.), and the Best Methods of apply- ing it in Ophthalmic Medicine. By D. IIanbury, F.L.S.-On the Genns Ceodes of Forster. By B. Seemann.-On the Toot-Poison of New Zealand. I3y W. L. Lindsay, M. D.-New Publications : Berkeley's 'IIandbook of British Mosses '; Caruel's 'Prodromo della Flora Toscana'; Preston's 'Flora of Marlborougl.'-Botanical News.

\section{PART IX.}

Revision of the Natural Order Bignoniacea. By B. Secmann. (With a Plate.)-On the Botany of South Pembrokeshirc. By C. C. Babington.-Report on the Royal Gardens at Kew. By Sir W. J. Honker, LI.J), etc. - On Iypericum lineolatum. 13y J. G. Baker. - On the Position of the Genera Hydrocotylc, Opa, Commia, and Blastus in the $\mathrm{Na}$ tural System. By 13. Seemann.-Rosa Iibernica, Sin.-Fumaria media, Lois.-Plants notieed at Hunstanton, on the Coast of Norfolk.-Fucus furcatus, Agardh: a New I3ritish Seaweed.-New Publications: 'Naturalists' Scrap-Book for the Liverpool District'; Boreau's 'Précis des principales Herborisations faites en Maine-et-Loire en 1862, suivi de dissertations critiques.'-Botanical News.

\section{PART $\mathrm{X}$}

On Mammillaria Scheerii, Mülllenpf., a Rare Mexiean Caetus. By B. Seemann. (With a Plate.) -On a Yorkshire Galium allied to G. erectum, Huds. By J. G. Baker.-Trichomanes radicans. -Comparison of the Leaf-cells in the British Hymenophyllex. By G Gnlliver, F.R.S.-Tithymalus Brauni, a New Euphorbiaeea from Abyssinia.-On Two Forms of Plants growing under the Same Conditions. By Dr. J. E. Gray, F.R.S. On the Origin of Herbaria. By Erust H. T. Meyer.-The Owala or Opacliala (Pentaclethra macropliylla, Benth.) of the Gaboon and Fernando Po, and the Oil containerl in its Seed. By J. Arnaudon. - New British Cryptogania.Chrysymenia rosea, Harv.-New Publieations: Brewer's 'Flora of Surrey '; Mueller's 'Fragmenta Phytographiæ Australiæ'; Rosenthal's 'Synopsis Plantarum Diaphoriearum'; 'Supplement to English Botany.'-Botanical News.

\section{PAR'T XI}

On the Geographical Distribution of the Equisetaceæ. By J. Milde, Ph.D. - Viola arenaria, De Cand., as a British Plant.-Goethe's Essay on the Metamorphosis of Plants.-Inflammability of the Flowers of Dictamnus albus.-Notes from Northumberland, Septem. 1863.-On the Natural Order Characeæ.-Cacoma piniquatorum, De Bory.-New Publications : Hardy's 'Liehen Flora of the Eastern Borders '; D. Moore's 'Contributions to the British and Irish Floras of Musei and Hepaticæ '; Carringtol1's 'Gleaniugs among the Irish Cryptogams.'Miscellanea: The Chinese Date Plum Acclinatized in New South Wales.-Botanical News.

\section{PART XII}

Fucus distichus, Linn., as an Irish Plant. By W. Carruthers.-Sagina nivalis, Fries, discovered in Seotland. By H. C. Watson, F.L.S.-Hypnum abietinum, Linn. By WV. Mitten, F.L.S.-Kemarks on the Yield of Quinine in the Leaves of Chinchona Plants. - By Dr. Anderson, F.L.S.-Is IIutchinsia alpina, R. Brown, a British Plant? By Rev. W W. Newbould, F.L.S.-Goethe's Essay on the Metamorphosis of Plants. - On the Nardoo Plant of Australia.-Chroolepus lageniferum, Hildebrand.Rare and Exotie Plants at Kew Bridge, Surrey.Stations of some Uneommon Plants in Deron and Cornwall.-New Publications: Prior's 'Popular Names of British Plants.'-Botanical News.

\section{LONDON : ROBERT HARDWICKE, 192, PICCADILLY;}

\section{Andrew Elliot, 15, Princes Street, Edinburg7; J. Rothschild, Paris;} L. Denicke, Leipzig; Westermann, New York. 


\section{LIS'I OF CONTRIBU'TORS}

TOLUME I. OF 'THE JOURNAL OF BOTANY.'

T. Anderson, M.D., F.L.S.

C. C. Babington, M.A., F.R.S., F.L.S.

J. G. Baker, Esq.

J. Ball, Esq.

G. Bennett, M.D., F.L.S.

J. J. Bennett, Esq., F.R.S., F.L.S.

A. A. Black, Esq.

T. R. A. Briggs, Esq.

J. Britten, Esq.

IV. Carruthers, Esq., F.L.S.

M. C. Cooke, Esq.

Miss E. M. Cox.

F. Currey, M.A., F.R.S., F.L.S.

M. Alphonse de Candolle.

H. R. Goeppert, Ph.D.

J. E. Gray, Ph.D., F.R.S., F.I.S.

G. Gulliver, Esq., F.R.S.

F. A. Hanbury, B.A.

D. Hanbury, Esq., F.L.S.

H. F. Hance, Ph.D.

J. E. Howard, Esq., F.L.S.

G. Hunt, Esq.

F. Leybold, M.D.

C. R. Markham, Esq., F.R.G.S.

M. T. Masters, M.D., F.L.S.

G. Maxwell, Esq.

J. Milde, Ph.D.

J. Miers, Esq., F.R.S., F.L.S.

W. Mitten, Esq., A.L.S. 
Thomas Moore, Esq., F.L.S.
A. G. More, Esq., F.L.S.
W. Mudd, Esq.
Rev. W. W. Newbould, M.A., F.L.S.
R. C. A. Prior, M.D., F.L.S.
II. Schott, Ph.D.
C. H. Schultz-Bipontinus, M.I).
J. Schweinfurth, Ph.D.
B. Seemann, Ph.D., F.L.S.
Alex. Smith, Esq.
J. T. Boswell Syme, Esq., F.L.S.
F. Townsend, M.A., F.L.S.
H. C. Watson, Esq., F.L.S.

The Journal of Botany is published on the 1st of every Month:

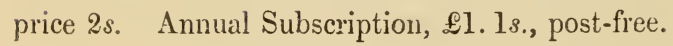

To Mr. R. Handwicke, 192, Piccadilly.

Please to send the Jounnal of Botany to the undermentioned addiess for One Year, for which I enclose One Guinea.

Name.

Address. 



UNIVERSITY OF ILLINOIS-URBANA 589.2 C77IN COO1

INDEX FUNGORUM BRITANNICORUMS LONDON

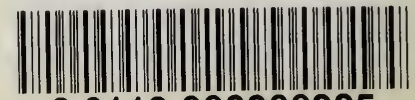

30112009980225 